\title{
Redshift-space correlation functions in large galaxy cluster surveys
}

\author{
P. Valageas ${ }^{1}$ and N. Clerc ${ }^{2}$ \\ ${ }^{1}$ Institut de Physique Théorique, CEA Saclay, 91191 Gif-sur-Yvette, France \\ e-mail: patrick.valageas@cea.fr \\ 2 Max-Planck-Institut für extraterrestrische Physik (MPE), Giessenbachstrasse 1, 85748 Garching, Germany
}

Received 22 May 2012 / Accepted 10 September 2012

\section{ABSTRACT}

\begin{abstract}
Context. Large ongoing and upcoming galaxy cluster surveys in the optical, X-ray and millimetric wavelengths will provide rich samples of galaxy clusters at unprecedented depths. One key observable for constraining cosmological models is the correlation function of these objects, measured through their spectroscopic redshift.

Aims. We study the redshift-space correlation functions of clusters of galaxies, averaged over finite redshift intervals, and their covariance matrices. Expanding as usual the angular anisotropy of the redshift-space correlation on Legendre polynomials, we consider the redshift-space distortions of the monopole as well as the next two multipoles, $2 \ell=2$ and 4 .

Methods. Taking into account the Kaiser effect, we developed an analytical formalism to obtain explicit expressions of all contributions to these mean correlations and covariance matrices. We include shot-noise and sample-variance effects as well as Gaussian and non-Gaussian contributions.

Results. We obtain a reasonable agreement with numerical simulations for the mean correlations and covariance matrices on large scales $\left(r>10 h^{-1} \mathrm{Mpc}\right)$. Redshift-space distortions amplify the monopole correlation by about $10-20 \%$, depending on the halo mass, but the signal-to-noise ratio remains of the same order as for the real-space correlation. This distortion will be significant for surveys such as DES, Erosita, and Euclid, which should also measure the quadrupole $2 \ell=2$. The third multipole, $2 \ell=4$, may only be marginally detected by Euclid.
\end{abstract}

Key words. surveys - galaxies: clusters: general - large-scale structure of Universe - cosmology: observations

\section{Introduction}

The growth of structure in our Universe results from a competition between gravitational attraction and the mean Hubble expansion. Studying recent structures provides an understanding of both these phenomena along with supplementary insights into the initial conditions that describe primordial density fluctuations, which are assumed to be nearly Gaussian-distributed. Analyses of massive large galaxy surveys (Cole et al. 2005; Moles et al. 2008; Eisenstein et al. 2011; Blake et al. 2011b), galaxy cluster surveys (Böhringer et al. 2001; Burenin et al. 2007; Koester et al. 2007a; Vanderlinde et al. 2010; Mehrtens et al. 2012), weak-lensing studies (e.g. Benjamin et al. 2007; Massey et al. 2007; Kilbinger et al. 2009; Schrabback et al. 2010), and measurements of the baryonic acoustic oscillation peak (Eisenstein et al. 2005; Blake et al. 2011a; Slosar et al. 2011) are currently under way or are planned to probe the physics of large scales in multiple complementary ways.

Clusters of galaxies are defined as galaxy concentrations, but they can also be regarded as a specific class of objects in their own right. In the hierarchical framework of structure formation, they are the largest and last nonlinear objects in a quasiequilibrium state. Observationally, they are well-defined and are systematically identified as strong X-ray emitters, thanks to their hot gas phase (e.g. Sarazin 1988; Pierre et al. 2011), and/or as isolated (red) galaxy overdensities (Abell 1958; Koester et al. 2007b), and/or via their imprint on the cosmological background radiation (Sunyaev \& Zeldovich 1972; Planck Collaboration 2011; Reichardt et al. 2012). Observed clusters are much more rare than individual galaxies (current cluster surveys amount up to a few tens of objects per $\mathrm{deg}^{2}$ ), but the link to their parent dark matter halo mass is better understood. Most of cluster cosmological studies rely on the abundance of clusters and its evolution (Borgani et al. 2001; Rozo et al. 2010; Mantz et al. 2010; Sehgal et al. 2011; Clerc et al. 2012). However, very large ongoing surveys (e.g. Pierre et al. 2011) or planned surveys (Predehl et al. 2009; Refregier et al. 2011) promise to overcome the statistical limit and better constrain cosmological models by stuyding the spatial clustering of clusters over large volumes.

This work follows our previous study (Valageas et al. 2011, hereafter Paper I) that analytically quantified covariance matrices for correlation functions in the framework of large galaxy cluster surveys. These calculations are essential to account for uncertainties in clustering analyses. Most generally, these uncertainties combine a shot-noise term (arising from the discreteness of the objects) and a sample variance term (due to the finite volume of the Universe being surveyed), as well as mixed contributions. All are related to the survey design and we applied our formalism to predict signal-to-noise ratios for observable quantities given different survey strategies (Pierre et al. 2011; Valageas et al. 2011). We presented our method in a way as close as possible to observational analyses, e.g. refering to the widely-used Landy-Szalay estimator for the two-point correlation function, integrated within fixed distance bins, averaging over all accessible redshift values, etc. Our results were found to agree well with large N-body simulations. However, only real-space comoving coordinates were considered, i.e., we ignored halo peculiar velocities. Because their radial component impacts object distance 
measurements via an additional spectral redshift, these peculiar velocities distort the correlation function estimates.

The goal of the present paper is to estimate the corrections induced by these redshift-space distortions, still considering galaxy clusters only, and to predict if these effects may be observed in present and future cluster surveys. Indeed, redshiftspace distortion measurements have attracted growing interest as a cosmological probe. Schematically, this can be understood as follows: because peculiar velocities are linked to the gravitational well created by surrounding matter, bulk flows in the large scale structure are directly linked to the growth rate of structures. More precisely, redshift-space distortions are sensitive to $D_{+}(a)$, the linear growth factor, as a function of cosmic scalefactor $a$, and to $f=\mathrm{d} \ln D_{+} / \mathrm{d} \ln a$. In particular, observational results have been obtained by various galaxy spectroscopic surveys such as the 2dFGRS (Peacock et al. 2001), the SDSS (Cabré \& Gaztañaga 2009; Reid et al. 2012), 2dF-SDSS LRG and QSO survey (Ross et al. 2007), and the VVDS Guzzo et al. (2008).

In the following, we assume galaxy clusters to have been detected individually in a dedicated survey (e.g. in the X-ray band) and their redshift to have been subsequently determined, e.g. by measurements of their member galaxy spectra and averaging over the corresponding galaxy redshifts. This is the most common - but observationally expensive - method to determine cluster redshifts. In practical applications, only a subsample of all cluster members can be observed due to observational constraints (limiting magnitude, compactness of the cluster in the instrumental focal plane, finite wavelength range of the spectrograph, identification of spectral lines, elimination of fore- and background objects, etc.). This translates into statistical errors on the cluster redshift estimate (e.g. Katgert et al. 1996; Adami \& Ulmer 2000). Most generally, spectroscopic redshifts of galaxy clusters are considered to be precise to $\sigma(z) \sim 0.01(1+z)$ or less.

To distinguish measurement errors from intrinsic covariances, we assume cluster redshifts to be known with infinite accuracy and neglect any kind of bias. Adding these sources of noise (which are not correlated with the shot-noise and samplevariance terms that we consider here) is not difficult as one only needs to add the relevant covariance matrices. We do not consider this second step here, which depends on the details of the instrument.

As in Paper I, we considered in our calculations the effects of shot-noise and sample variance. We expanded the angular anisotropy of the redshift-space correlation function on Legendre polynomials. Our formalism then leads to expressions for the monopole and the two multipoles $2 \ell=2$ and $2 \ell=4$. We provide explicit expressions for the expected quantities and their covariance matrices, taking into account the Kaiser effect and Gaussian as well as non-Gaussian contributions. Keeping in mind the application to galaxy cluster surveys, all quantities were integrated within the $0<z<1$ redshift range. Halos were defined by their mass $M$ and we describe the population by using a mass function and a bias model from previous works. The three- and four-point correlation function were estimated by means of a hierarchical model (Peebles 1980; Bernstein 1994), where those quantities only depend on combinations of twopoint correlation functions. Comparisons with numerical simulations were made to assess the reliability of our analytical calculations.

Therefore, our main improvements over previous works consist in the inclusion of Gaussian and non-Gaussian contributions in the computation of covariance matrices, as well as in the derivation of observables close to those expected in practical studies focused on galaxy clusters.
This paper is organized as follows. In Sect. 2 we first briefly describe the analytic models that we used to estimate the means and covariance matrices of redshift-space halo correlation functions, as well as the numerical simulations that we used to check our results. Then, focusing on the Landy \& Szalay estimator, we study the means of the various multipoles of the redshift-space correlation in Sect. 3, and their covariance matrices in Sect. 4. We compare our analytical results with numerical simulations for the two lowest multipoles (the " $2 \ell=0$ " monopole and the " $2 \ell=2$ " quadrupole). We apply our formalism to several real surveys in Sect. 5, for the three multipoles generated by the Kaiser effect ( $2 \ell=0,2$, and 4). We conclude in Sect. 6.

We give details of our calculations in Appendices A and $\mathrm{B}$, and we show the auto- and cross-correlation matrices of the " $2 \ell=2$ " multipole in Appendix C.

\section{Redshift-space halo density field}

Before we describe our analysis of the covariance matrices for halo number counts and correlation functions, we present in this section the analytic models that we used for the underlying halo distributions (mass function and bias, etc.) and the numerical simulations that we used to validate our results.

\subsection{Analytic models}

To be consistent with the numerical simulations, we use the WMAP3 cosmology in Sects. 3 and 4 where we develop our formalism and compare our results with simulations, that is, $\Omega_{\mathrm{m}}=0.24, \Omega_{\mathrm{de}}=0.76, \Omega_{b}=0.042, h=0.73, \sigma_{8}=0.77$, $n_{\mathrm{s}}=0.958$, and $w_{\mathrm{de}}=-1$ (Spergel et al. 2007). In Sect. 5, where we apply our formalism to obtain forecasts for current and future surveys, we use the more recent WMAP7 cosmology (Komatsu et al. 2011), that is, $\Omega_{\mathrm{m}}=0.274, \Omega_{\mathrm{de}}=0.726, \Omega_{b}=0.046$, $h=0.702, \sigma_{8}=0.816, n_{\mathrm{s}}=0.968$, and $w_{\mathrm{de}}=-1$.

Keeping in mind the study of X-ray clusters, we considered the number counts and correlations of dark matter halos defined by the nonlinear density contrast $\delta=200$. These halos are fully characterized by their mass, and we did not investigate the relationship between this mass and cluster properties such as the gas temperature and X-ray luminosity. These scaling laws can be added to our formalism to derive the cluster number counts and correlations, depending on the quantities that are actually measured, but we kept a more general setting in this paper.

\subsubsection{Halo mass function and bias}

We use the halo mass function, $\mathrm{d} n / \mathrm{d} \ln M$, of Tinker et al. (2008), and the halo bias, $b(M)$, of Tinker et al. (2010). Then, we write the two-point real-space correlation function between two halos labeled " $i$ " and " $j$ " as

$\xi_{i, j}^{\mathrm{h}}=b_{i} b_{j} \xi\left(\left|\boldsymbol{x}_{i}-\boldsymbol{x}_{j}\right| ; z\right)$,

where $\xi$ is the real-space matter density correlation. This corresponds to the halo power spectrum

$P_{i, j}^{\mathrm{h}}(k ; z)=b_{i} b_{j} P(k ; z)$,

where $P(k)$ is the matter density power spectrum. Here we used the approximation of scale-independent halo bias, which is valid to better than $10 \%$ on scales $20<x<130 h^{-1}$ Mpc (Manera \& Gaztanaga 2011), with a small feature on the baryon acoustic scale $\left(r \sim 100 h^{-1} \mathrm{Mpc}\right)$ of amplitude of 5\% (Desjacques et al. 2010). 
To simplify notations, we define the mean cumulative number density $\bar{n}$ of objects observed at a given redshift, within the mass bin $\left[M_{-}, M_{+}\right]$, as

$\bar{n}(z)=\int_{M_{-}}^{M_{+}} \frac{\mathrm{d} M}{M} \frac{\mathrm{d} n}{\mathrm{~d} \ln M}(M, z)$,

and the mean bias $\bar{b}$ as

$\bar{b}(z) \bar{n}(z)=\int_{M_{-}}^{M_{+}} \frac{\mathrm{d} M}{M} b(M, z) \frac{\mathrm{d} n}{\mathrm{~d} \ln M}(M, z)$.

In the following we omit to write the explicit boundaries on mass since we only consider a single mass bin. It is straightforward to extend all our results to the case of several mass bins by writing the relevant mass boundaries and replacing factors such as $\bar{b}^{2} \bar{n}^{2}$ by $\bar{b}_{i} \bar{n}_{i} \bar{b}_{j} \bar{n}_{j}$.

\subsubsection{Redshift-space correlation functions}

We denote by $\boldsymbol{x}$ and $\boldsymbol{s}$ real-space and redshift-space coordinates. They only differ by their radial component because of the peculiar velocity along the line-of-sight,

$\boldsymbol{s}=\boldsymbol{x}+\frac{\boldsymbol{e}_{z} \cdot \boldsymbol{v}}{a H} \boldsymbol{e}_{z}$

Here, $\boldsymbol{e}_{z}$ is the unit vector along the line of sight and $\boldsymbol{v}$ the peculiar velocity. At linear order, the peculiar velocity field and the density contrast are related in Fourier space as

$\tilde{\boldsymbol{v}}(\boldsymbol{k})=\mathrm{i} a \frac{\mathrm{d} \ln D_{+}}{\mathrm{d} t} \frac{\boldsymbol{k}}{k^{2}} \tilde{\delta}(\boldsymbol{k})$

where $D_{+}$is the linear growing mode. From the conservation of matter, $\left(1+\delta^{(s)}\right) \mathrm{d} \boldsymbol{s}=(1+\delta) \mathrm{d} \boldsymbol{x}$, one obtains the redshift-space matter power spectrum (Kaiser 1987)

$P^{(s)}(\boldsymbol{k} ; z)=\left(1+f \mu_{\boldsymbol{k}}^{2}\right)^{2} P(k ; z), \quad \mu_{k}=\frac{\boldsymbol{e}_{z} \cdot \boldsymbol{k}}{k}$,

where

$f=\frac{\mathrm{d} \ln D_{+}}{\mathrm{d} \ln a}$.

Here and in the following, we use a superscript $(s)$ for redshiftspace quantities. Following common practice, we assume that the halo velocity field is not biased. This yields at the same order the redshift-space halo power spectrum

$P^{\mathrm{h}(s)}(\boldsymbol{k} ; z)=\bar{b}^{2}\left(1+\beta \mu_{\boldsymbol{k}}^{2}\right)^{2} P(k ; z)$,

with $\beta=f / \bar{b}$, where $\bar{b}$ is the mean bias of the halo population as defined in Eq. (4) (Percival \& White 2009). We write the associated redshift-space halo correlation function as

$\xi^{\mathrm{h}(s)}(s ; z)=\bar{b}^{2} \xi^{(s)}(s ; z)$,

where $\xi^{(s)}(s)$ can be expanded as (Hamilton 1992)

$\xi^{(s)}(s)=\sum_{\ell=0}^{2} \xi^{(s ; 2 \ell)}(s) P_{2 \ell}\left(\mu_{\boldsymbol{s}}\right), \quad \mu_{\boldsymbol{s}}=\frac{\boldsymbol{e}_{z} \cdot \boldsymbol{s}}{s}$, where we introduced the functions

$$
\begin{aligned}
& \xi^{(s ; 0)}(s)=\left(1+\frac{2 \beta}{3}+\frac{\beta^{2}}{5}\right) \int_{0}^{\infty} \frac{\mathrm{d} k}{k} \Delta^{2}(k) j_{0}(k s), \\
& \xi^{(s ; 2)}(s)=-\left(\frac{4 \beta}{3}+\frac{4 \beta^{2}}{7}\right) \int_{0}^{\infty} \frac{\mathrm{d} k}{k} \Delta^{2}(k) j_{2}(k s), \\
& \xi^{(s ; 4)}(s)=\frac{8 \beta^{2}}{35} \int_{0}^{\infty} \frac{\mathrm{d} k}{k} \Delta^{2}(k) j_{4}(k s),
\end{aligned}
$$

where $j_{\ell}$ are the spherical Bessel functions and we introduced the logarithmic power $\Delta^{2}(k)=4 \pi k^{3} P(k)$.

The function $\xi^{(s)}$ and its multipoles $\xi^{(s ; 2 \ell)}$ depend on the halo population through the factor $\beta$ (i.e., the bias cannot be fully factored out as in the real-space correlation (1)). However, it is convenient to introduce the auxiliary function $\xi^{(s)}$ as in Eq. (10) so that most expressions obtained in the following have the same form as in real space (which is recovered by setting $\beta=0$, that is, $\left.\xi^{(s)}=\xi\right)$.

Independently of the linear-order approximation (9), only even multipoles can appear in the expansion (11) because of parity symmetry in redshift-space. Nonlinear corrections may add higher-order even multipoles that could be handled by a straightforward generalization of the approach developed in this paper.

The redshift-space distortions (9) and (11) only include the "Kaiser effect" (Kaiser 1987), associated with large-scale flows (i.e., the collapse of overdense regions and the expansion of underdense regions), which amplifies the power because the density and velocity fields are closely related as in Eq. (6). However, on smaller scales within virialized regions, there appears a high velocity dispersion that adds a decorrelated random component to relation (5). This yields the so-called "fingers of God" (Jackson 1972) that can be seen on galaxy surveys and give a damping factor to their redshift-space power spectrum. In this paper we focus on cluster surveys where this effect does not arise (as can be checked in numerical simulations, Nishimichi \& Taruya 2011). Indeed, because clusters are the largest nonlinear objects, cluster pairs are still in the process of moving closer (or farther appart in fewer cases) and have not had time to fall within a common potential well and develop a high velocity dispersion through several orbital revolutions. Therefore, we only need to consider the Kaiser effect as in Eqs. (9) and (11). This provides a good agreement with numerical simulations for $s>20 h^{-1} \mathrm{Mpc}$ (within 10\%, except for the multipole $2 \ell=4$, which shows stronger deviations from linear theory, Reid \& White (2011), but we will see that this multipole is not very important for cluster studies), and for $k<0.1 \mathrm{~h} \mathrm{Mpc}^{-1}$ in Fourier space (Percival \& White 2009; Nishimichi \& Taruya 2011).

We assume that these expressions still provide a good approximation up to the weakly nonlinear scales that are relevant for studies of cluster correlations $\left(z 6 h^{-1} \mathrm{Mpc}\right)$. For numerical purposes we use the popular fitting formula to numerical simulations of Smith et al. (2003) for the matter power spectrum.

Nevertheless, the formalism that we develop in the following sections is fully general and does not rely on Eqs. (12)-(14) and the truncation of the series (11) at $2 \ell=4$. It can be applied to any model for the redshift-space correlation $\xi^{(s)}(s)$. If this leads to higher-order multipoles $\xi^{(s ; 2 \ell)}$, one simply needs to extend the sums to all required multipoles in the various expressions given in Appendix A.

\subsubsection{Three-point and four-point halo correlations}

The covariance matrices of estimators of the halo two-point correlation involve the three-point and four-point halo correlations. 
Therefore, we need a model for these higher-order correlations. As in Valageas et al. (2011), following Bernstein (1994), Szapudi \& Colombi (1996), and Meiksin \& White (1999), we use a hierarchical ansatz (Groth \& Peebles 1977; Peebles 1980) and we write the real-space halo three-point correlation $\zeta^{\mathrm{h}}$ as

$\zeta_{1,2,3}^{\mathrm{h}}=b_{1} b_{2} b_{3} \frac{S_{3}}{3}\left[\xi_{1,2} \xi_{1,3}+\xi_{2,1} \xi_{2,3}+\xi_{3,1} \xi_{3,2}\right]$,

where we sum over all three possible configurations over the three halos labeled " 1 ", " 2 ", and " 3 ", and the real-space halo four-point correlation $\eta^{\mathrm{h}}$ as

$$
\begin{aligned}
\eta_{1,2,3,4}^{\mathrm{h}}= & b_{1} b_{2} b_{3} b_{4} \frac{S_{4}}{16}\left[\xi_{1,2} \xi_{1,3} \xi_{1,4}+3\right. \text { cyc. } \\
& \left.+\xi_{1,2} \xi_{2,3} \xi_{3,4}+11 \text { cyc. }\right]
\end{aligned}
$$

where " 3 cyc." and "11 cyc." stand for three and eleven terms that are obtained from the previous one by permutations over the labels "1, 2, 3, 4" of the four halos. As in Valageas et al. (2011), we take for the normalization factors $S_{3}$ and $S_{4}$ their large-scale limit, which is obtained by perturbation theory (Peebles 1980; Fry 1984; Bernardeau et al. 2002b),

$S_{3}=\frac{34}{7}-(n+3)$,
$S_{4}=\frac{60712}{1323}-\frac{62}{3}(n+3)+\frac{7}{3}(n+3)^{2}$,

where $n$ is the slope of the linear power spectrum at the scale of interest. The model (15)-(18) is the simplest model that qualitatively agrees with large-scale theoretical predictions (which also give the scalings $\zeta \sim \xi^{2}$ and $\eta \sim \xi^{3}$, but where the normalization factors $S_{3}$ and $S_{4}$ show an additional angular dependence) and small-scale numerical results (Colombi et al. 1996) (which however show an additional moderate scale-dependence of the normalization factors $S_{3}$ and $S_{4}$ ). As shown in Valageas et al. (2011), this model (15)-(18) provides reasonably good predictions for the covariance matrices of estimators of the cluster twopoint correlations (which focus on weakly nonlinear scales).

Next, for the redshift-space correlations $\zeta^{\mathrm{h}(s)}$ and $\eta^{\mathrm{h}(s)}$ we use the same hierarchical ansatz (15)-(16), where in the righthand-side we replace $\xi$ by the redshift-space two-point correlation $\xi^{(s)}$ given in Eq. (11). This redshift-space model has not been checked in details against numerical simulations in previous works and we compare our predictions with simulations in Sect. 4 below. We will check that it yields a reasonable agreement for covariance matrices (especially along the diagonal) as for our purposes we only need a simple and efficient approximation. If one aims at predicting the three-point and four-point redshift-space correlations for their own sake, up to a high accuracy, one should perform dedicated tests for these quantities and probably build more sophisticated models, but this is beyond the scope of this paper.

This approximation allows us to extend in a straightforward fashion the formalism developed in Valageas et al. (2011) to redshift-space estimators. The only difference comes from the angular dependence of the two-point correlation (11), that is, from the second and fourth spherical harmonics $\xi^{(s ; 2)}(s) P_{2}(\mu)$ and $\xi^{(s ; 4)}(s) P_{4}(\mu)$. In particular, as clearly seen in Eq. (9), by setting $\beta=0$, which only leaves the monopole contribution $\ell=0$, we recover the real-space results given in Valageas et al. (2011).

\subsection{Numerical simulations}

To check our analytical model, we compare our predictions with the numerical simulations that we used in Valageas et al. (2011) for real-space statistics. While we had considered only realspace coordinates in our previous study, we used the peculiar velocity assigned to each halo to conduct our comparison in redshift-space coordinates.

Here we briefly recall the main characteristics of these simulations. We used the high-resolution, all-sky, "horizon" simulation run (Teyssier et al. 2009). It consists of a $\left(2 h^{-1} \mathrm{Gpc}\right)^{3}$ $\mathrm{N}$-body simulation carried out with the RAMSES code (Teyssier 2002). The effect of baryons is neglected and the total number of particles in the simulation sets the total mass of the simulation. The mass resolution, which is the mass of each of the $4096^{3}$ simulated particules, is $\sim 10^{10} M_{\odot}$. Halos are found using the HOP algorithm (Eisenstein \& Hut 1998). The (real-space) comoving position and peculiar velocity of each halo are obtained by averaging over all particles making up its total mass. Comoving coordinates are further transformed into sky positions (two angles plus redshift) and redshift-space coordinates taking into account their radial velocity. To ensure completeness of the simulation in all directions, we only considered halos at $z<0.8$.

Simulated surveys are extracted from the full-sky light-cone catalog by defining non-overlapping rectangular regions in angular coordinates. Each field covers an area of $50 \mathrm{deg}^{2}$. To minimize correlations between neighboring fields, we required a separation of 20 deg between two consecutive surveys, which results in a total of 34 such independent fields distributed all across the sky. Only for the results discussed in Sect. 4.3 we also used a $10 \mathrm{deg}$ gap (resulting in 138 fields). For the purpose of deriving the correlation function, auxiliary random fields were created by shuffling the angular coordinates of halos in the original data fields. This operation preserves the original redshift and mass distribution of the simulation. The density of objects in the random fields was increased to one hundred times that of original data fields to avoid introducing spurious noise. The LandySzalay estimator and its generalization (see Sect. 3.1, Eq. (20)) provide an estimate of the two-point correlation and its multipoles in redshift space. As in Valageas et al. (2011), the mean and covariance of all derived quantities were finally estimated by sample averaging over the extracted fields.

\section{Mean redshift-space two-point correlation functions}

Since we have in mind the application to cluster surveys and, more generally, to deep surveys of rare objects, we considered 3D correlation functions averaged over a wide redshift bin (to accumulate a large enough number of objects), rather than the usual local 3D correlation functions at a given redshift. This means that the quantities that we considered, while being truly $3 \mathrm{D}$ correlations and not 2D angular correlations, nevertheless involve integrations along the line of sight within a finite redshift interval. We followed the formalism and the notations of Valageas et al. (2011) to derive the means and the covariance matrices of estimators of these integrated redshift-space correlations.

\subsection{Analytical results}

We focus on the Landy \& Szalay estimator of the two-point correlation Landy \& Szalay (1993), given by

$\hat{\xi}=\frac{D D-2 D R+R R}{R R}$,

where $D$ represents the data field and $R$ an independent Poisson distribution, both with the same mean density. In practice, before 
appropriate rescaling, the mean number density of the Poisson process $R$ is actually taken to be much higher than the observed one, so that the contribution from fluctuations of the denominator $R R$ to the noise of $\hat{\xi}$ can be ignored. The advantage of Eq. (19) is that one automatically includes the geometry of the survey (including boundary effects, cuts, etc.), because the auxiliary field $R$ is drawn on the same geometry. In practice, our generalization of the estimator (19) to redshift-space multipoles writes as

$$
\begin{aligned}
\hat{\xi}_{R_{i}, 2 \ell_{i}}^{(s)}= & \frac{4 \ell_{i}+1}{\sum_{r r} 1}\left\{\sum_{d d} P_{2 \ell_{i}}\left(\mu_{d d}\right)-2 \sum_{d r} P_{2 \ell_{i}}\left(\mu_{d r}\right)\right. \\
& \left.+\sum_{r r} P_{2 \ell_{i}}\left(\mu_{r r}\right)\right\},
\end{aligned}
$$

where each sum counts all the pairs, $d d, d r$, or $r r$, within the samples $D$ and $R$, that are separated by a redshift-space distance $s$ within the bin $R_{i,-}<s<R_{i,+}$. Compared with the usual monopole estimator (19), we added the geometrical weight $\left(4 \ell_{i}+1\right) P_{2 \ell_{i}}(\mu)$ to each pair in the numerator while in the denominator we kept the unit weight. Of course, for $\ell_{i}=0$ we recover Eq. (19).

Within our framework, we write this estimator $\hat{\xi}_{R_{i}, 2 \ell_{i}}^{(s)}$, for the multipole $2 \ell_{i}$ of the mean correlation on scales delimited by $R_{i,-}$ and $R_{i,+}$, integrated over some redshift range and mass interval, as

$$
\begin{aligned}
& \hat{\xi}_{R_{i}, 2 \ell_{i}}^{(s)}=\frac{1}{Q_{i}} \int \mathrm{d} z \frac{\mathrm{d} \chi}{\mathrm{d} z} \mathcal{D}^{2} \int \frac{\mathrm{d} \Omega}{(\Delta \Omega)} \int \frac{\mathrm{d} M}{M} \int_{i} \mathrm{~d} s^{\prime}\left(4 \ell_{i}+1\right) P_{2 \ell_{i}}\left(\mu^{\prime}\right) \\
& \quad \times \int \frac{\mathrm{d} M^{\prime}}{M^{\prime}} \frac{\mathrm{d} \hat{n}}{\mathrm{~d} \ln M} \frac{\mathrm{d} \hat{n}}{\mathrm{~d} \ln M^{\prime}}-\frac{2}{Q_{i}} \int \mathrm{d} z \frac{\mathrm{d} \chi}{\mathrm{d} z} \mathcal{D}^{2} \int \frac{\mathrm{d} \Omega}{(\Delta \Omega)} \int \frac{\mathrm{d} M}{M} \\
& \quad \times \int_{i} \mathrm{~d} s^{\prime}\left(4 \ell_{i}+1\right) P_{2 \ell_{i}}\left(\mu^{\prime}\right) \int \frac{\mathrm{d} M^{\prime}}{M^{\prime}} \frac{\mathrm{d} \hat{n}}{\mathrm{~d} \ln M} \frac{\mathrm{d} n}{\mathrm{~d} \ln M^{\prime}}+\delta_{\ell_{i}, 0},
\end{aligned}
$$

with

$$
\begin{aligned}
Q_{i}= & \int \mathrm{d} z \frac{\mathrm{d} \chi}{\mathrm{d} z} \mathcal{D}^{2} \int \frac{\mathrm{d} \Omega}{(\Delta \Omega)} \int \frac{\mathrm{d} M}{M} \int_{i} \mathrm{~d} \boldsymbol{s}^{\prime} \int \frac{\mathrm{d} M^{\prime}}{M^{\prime}} \\
& \times \frac{\mathrm{d} n}{\mathrm{~d} \ln M} \frac{\mathrm{d} n}{\operatorname{dln} M^{\prime}} .
\end{aligned}
$$

Here we denoted $\int_{i} \mathrm{~d} \boldsymbol{s}^{\prime}$ as the integral $\int_{R_{i,-}}^{R_{i,+}} \mathrm{d} \boldsymbol{s}^{\prime}$ over the 3D spherical shell $i$, of inner and outer radii $R_{i,-}$ and $R_{i,+}$ in redshift-space, $\mu^{\prime}=\left(\boldsymbol{e}_{z} \cdot \boldsymbol{s}^{\prime}\right) / s^{\prime}$ is the cosine of the angle between the line of sight and the halo pair, $\chi(z)$ and $\mathcal{D}(z)$ are the comoving radial and angular distances, $\delta_{\ell_{i}, 0}$ is the Kronecker symbol (which is unity if $\ell_{i}=0$ and zero otherwise), and $\mathrm{d} \hat{n} / \mathrm{d} \ln M$ is the observed density of objects. Here and in the following, we note observed quantities by a hat (i.e., in one realization of the sky) to distinguish them from mean quantities, such as the mean comoving number density $\mathrm{d} n / \mathrm{d} \ln M$. The two subscripts $R_{i}$ and $2 \ell_{i}$ label the radial bin and the multipole. If we consider $N_{R}$ radial bins and $N_{\ell}$ multipoles, there are $N_{R} \times N_{\ell}$ values of the index $i$ (i.e., two different indices $i$ and $j$ may correspond to different radial bins and multipoles, or to the same radial bin but with two different multipoles, and vice versa).

In Eqs. (21) and (22) we considered a survey with a single window of angular area $(\Delta \Omega)$ and a single mass bin. It is straigtforward to generalize to several distant (uncorrelated) angular windows and to several mass bins. The redshift interval $\Delta z$ is not necessarily small, and to increase the statistics we can choose the whole redshift range of the survey. It is again straigtforward to generalize to several nonoverlapping redshift intervals, if they are large enough to neglect cross-correlations between different bins ( $\Delta z \gtrsim 0.1$, see Fig. 11 in Valageas et al. 2011).
As in Eq. (20), the counting method that underlies the first term in Eq. (21) can be understood as follows. We span all objects in the "volume" $(z, \boldsymbol{\Omega}, \ln M)$, and count all neighbors at distance $s^{\prime}$, within the shell $\left[R_{i,-}, R_{i,+}\right]$, with a mass $M^{\prime}$. We denote with unprimed letters the quantities associated with the first object, $(z, \boldsymbol{\Omega}, \ln M)$, and with primed letters the quantities associated with the neighbor of mass $M^{\prime}$ at distance $s^{\prime}$. Thus, with obvious notations, $\mathrm{d} \hat{n} / \mathrm{d} \ln M$ and $\mathrm{d} \hat{n} / \mathrm{d} \ln M^{\prime}$ are the observed number densities at the first and second (neighboring) points. In contrast, the denominator $Q_{i}$ involves the mean number densities $\mathrm{d} n / \mathrm{d} \ln M$ and $\mathrm{d} n / \mathrm{d} \ln M^{\prime}$. Therefore, $Q_{i}$ is not a random quantity and accordingly it shows no noise. Similarly, the difference between the terms associated with $D D$ and $D R$ is that in the former we have a product of two observed number densities, $(\mathrm{d} \hat{n} / \mathrm{d} \ln M) \times\left(\mathrm{d} \hat{n} / \mathrm{d} \ln M^{\prime}\right)$, while in the latter we have a crossproduct between the observed and the mean number densities, $(\mathrm{d} \hat{n} / \mathrm{d} \ln M) \times\left(\mathrm{d} n / \mathrm{d} \ln M^{\prime}\right)$.

Then, if $\ell_{i} \neq 0$ the second and third terms in Eq. (21) vanish. This also means that for $\ell_{i} \neq 0$ the Landy \& Szalay estimator (20) and the Peebles \& Hauser estimator (Peebles \& Hauser 1974), which would read as $\hat{\xi}=D D / R R-\delta_{\ell_{i}, 0}$ (with the implicit factor $\left(4 \ell_{i}+1\right) P_{2 \ell_{i}}$ in $\left.D D\right)$, are equivalent (in the limit where the density of the auxiliary random sample $R$ goes to infinity).

The angular factor $\left(4 \ell_{i}+1\right) P_{2 \ell_{i}}\left(\mu^{\prime}\right)$ ensures that the estimator (21) extracts the multipole $2 \ell_{i}$ of the redshift-space correlation, as in (11). In our previous analysis of the real-space correlation (Valageas et al. 2011) we only needed to consider the monopole term, $\ell_{i}=0$, because higher multipoles vanished. Here, because the redshift-space correlation is anisotropic, higher multipoles are nonzero and contain valuable information. As seen in Eqs. (12)-(14), they constrain the parameter $\beta=f / \bar{b}$, and in turn the growth rate of the density fluctuations and the bias of the halos.

The quantity $Q_{i}$ also writes as ${ }^{1}$

$Q_{i}=\int \mathrm{d} \chi \mathcal{D}^{2} \bar{n}^{2} \mathcal{V}_{i}$

where the volume $\mathcal{V}_{i}$ of the $i$-shell is

$\mathcal{V}_{i}(z)=\frac{4 \pi}{3}\left[R_{i,+}(z)^{3}-R_{i,-}(z)^{3}\right]$,

which may depend on $z$. In practice, one would usually choose constant comoving shells, so that $\mathcal{V}_{i}$ does not depend on $z$. To obtain Eq. (23) we used that $\mathrm{d} n / \mathrm{d} \ln M$ and $\mathrm{d} n / \mathrm{d} \ln M^{\prime}$ have no scale dependence (because they correspond to a uniform distribution of objects) and we neglected edge effects. (These finite-size effects are discussed and evaluated in Appendix B of Valageas et al. 2011). Next, the mean of the estimator $\hat{\xi}^{(s)}$ reads as

$\left\langle\hat{\xi}_{R_{i}, 2 \ell_{i}}^{(s)}\right\rangle=\frac{1}{Q_{i}} \int \mathrm{d} \chi \mathcal{D}^{2} \bar{b}^{2} \bar{n}^{2} \mathcal{V}_{i} \overline{\xi_{R_{i}, 2 \ell_{i}}^{(s)}}(z)$,

with

$\overline{\xi_{R_{i}, 2 \ell_{i}}^{(s)}}(z)=\int_{i} \frac{\mathrm{d} s^{\prime}}{\mathcal{V}_{i}}\left(4 \ell_{i}+1\right) P_{2 \ell_{i}}\left(\mu^{\prime}\right) \xi^{(s)}\left(s^{\prime} ; z\right)$,

1 We can neglect the impact of the change from $x$ to $s$ on background cosmological quantities such as the radial and angular distances $\chi$ and $\mathcal{D}$, which vary on scales on the order of $c / H_{0}$. Redshift-space distortions only have a significant effect on the correlation $\xi^{(s)}$ of the halo population, which is studied on smaller scales $\sim 20 h^{-1}$ Mpc and becomes anisotropic. 


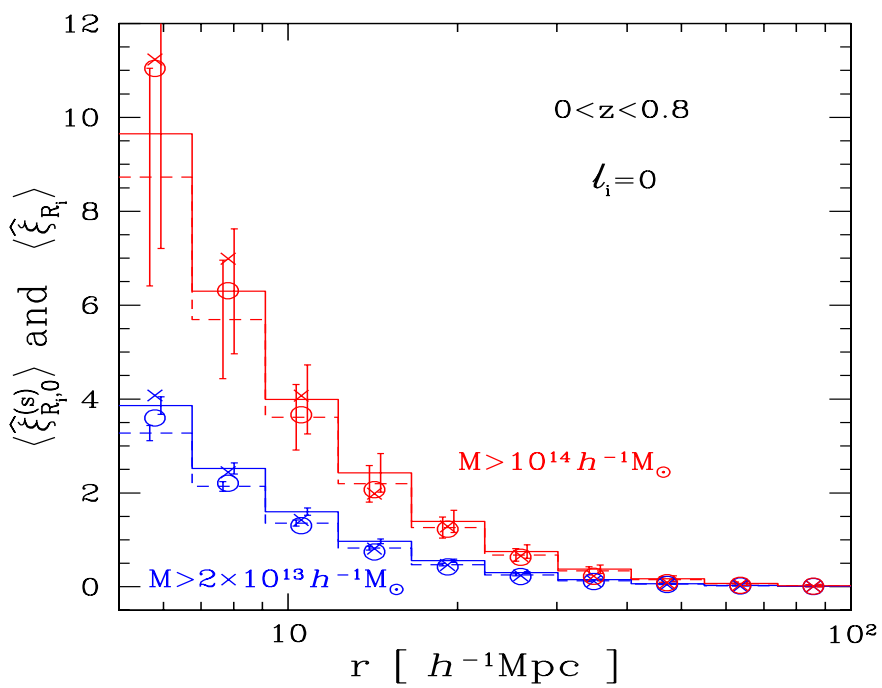

Fig. 1. Mean redshift-space (solid lines) and real-space (dashed lines) halo correlations, $\left\langle\hat{\xi}_{R_{i}, 0}^{(s)}\right\rangle$ and $\left\langle\hat{\xi}_{R_{i}}\right\rangle$. We considered ten comoving distance bins, within $5<r<100 h^{-1} \mathrm{Mpc}$, equally spaced in $\log (r)$. We counted all halos above the thresholds $M_{*}=2 \times 10^{13}$ and $10^{14} h^{-1} M_{\odot}$, within the redshift interval $0<z<0.8$. We compare our analytical results with numerical simulations (crosses for the monopole redshiftspace correlation and circles for the real-space correlation). The $3-\sigma$ error bars (slightly shifted to the left and to the right for the real-space and redshift-space correlations) are obtained from the analytical covariance matrices derived in Sect. 4.

which is the radial average of the $2 \ell_{i}$-multipole of $\xi^{(s)}$, over the 3D spherical shell associated with the radial bin $i$ in redshift space. Indeed, from the multipole expansion (11) this also reads as

$\overline{\xi_{R_{i}, 2 \ell_{i}}^{(s)}}=\int_{R_{i,-}}^{R_{i,+}} \frac{\mathrm{d} r 3 r^{2}}{R_{i,+}^{3}-R_{i,-}^{3}} \xi^{\left(s ; 2 \ell_{i}\right)}(r)$.

\subsection{Comparison with simulations}

We show in Fig. 1 the mean redshift-space and real-space halo correlations, $\left\langle\hat{\xi}_{R_{i}, 0}^{(s)}\right\rangle$ and $\left\langle\hat{\xi}_{R_{i}}\right\rangle$, over ten distance bins. We find that because of the prefactor $\left(1+2 \beta / 3+\beta^{2} / 5\right)$ in Eq. (12) the monopole redshift-space correlation is greater than the realspace correlation by $10 \%$ for massive halos, $M>10^{14} h^{-1} M_{\odot}$, and by $18 \%$ for $M>2 \times 10^{13} h^{-1} M_{\odot}$, in the redshift range $0<z<0.8$. The relative redshift-space distortion is weaker for more massive halos because they have a larger bias $\bar{b}$. This decreases the factor $\beta=f / \bar{b}$.

Although the results from numerical simulations are somewhat noisy, they agree reasonably well with our analytical predictions. They give the same order of magnitude as our predictions for both the redshift-space and real-space correlations. In particular, they fall within the expected $3-\sigma$ error-bars from our analytical predictions (these error bars are obtained from the analytical covariance matrices derived in Sect. 4 below). This also shows that the accurate computation of such quantities remains a difficult task for numerical simulations (in this case it would require many realizations of the simulated sky resulting in a heavy computational load) and it is useful to have analytical models for cross-checking. In particular, while the ratio $\left\langle\hat{\xi}_{R_{i}, 0}^{(s)}\right\rangle /\left\langle\hat{\xi}_{R_{i}}\right\rangle$ is greater than unity and smooth within the analytical model (it is

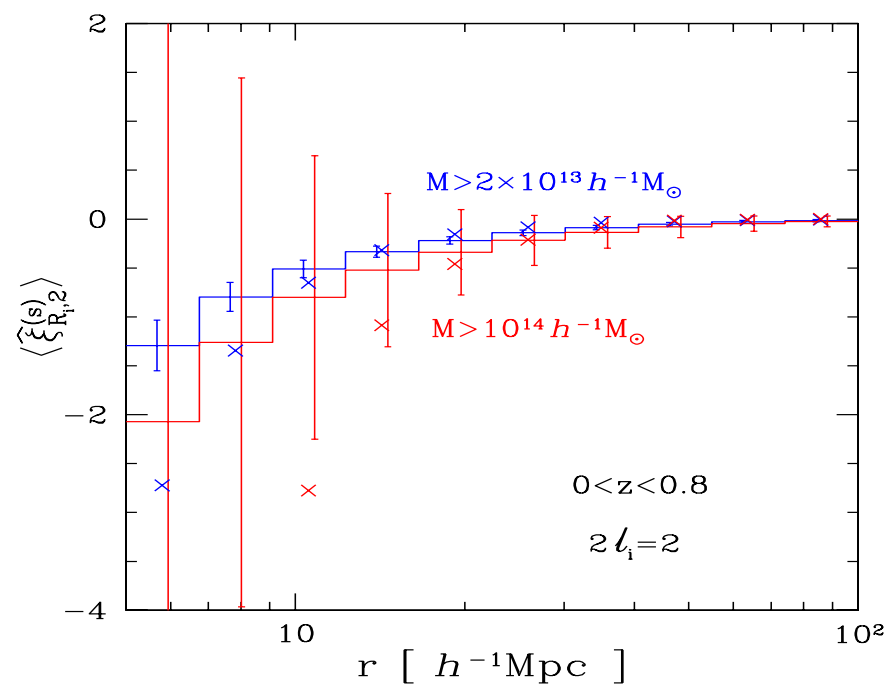

Fig. 2. " $2 \ell=2$ " redshift-space halo correlation $\left\langle\hat{\xi}_{R_{i}, 2}^{(s)}\right\rangle$. As in Fig. 1, we considered ten comoving distance bins, within $5<r<100 h^{-1} \mathrm{Mpc}$, equally spaced in $\log (r)$, and counted all halos above the thresholds $M_{*}=2 \times 10^{13}$ and $10^{14} h^{-1} M_{\odot}$, within the redshift interval $0<z<0.8$. We compare our analytical results with numerical simulations (crosses). The $3-\sigma$ error bars (slightly shifted to the left and to the right for the real-space and redshift-space correlations) are obtained from the analytical covariance matrices derived in Sect. 4.

actually scale-independent in our simple model (12)), it shows spurious fluctuations in the simulation data for rare massive halos because of low statistics.

For practical purposes, Fig. 1 shows that to constrain cosmology with the clustering of X-ray clusters it is necessary to take into account redshift-space distortions if one aims at an accuracy better than $10 \%$.

We show in Fig. 2 our results for the " $2 \ell=2$ " redshift-space correlation $\xi_{R_{i}, 2}^{(s)}$. This corresponds to the second term of the expansion (11) over Legendre polynomials. As seen from Eq. (13), this term is negative and its amplitude is larger for more massive halos because of the factor $\bar{b}^{2}\left(4 \beta / 3+4 \beta^{2} / 7\right)=4 f \bar{b} / 3+4 f^{2} / 7$, which grows with the halo bias. However, because the error bars are much larger for rare massive halos, the associated signal is more difficult to measure. We obtain a good agreement with the simulations on large scales but on small scales below $10 h^{-1}$ Mpc our model seems to underestimate the amplitude of this quadrupole component of the redshift-space correlation. This may be partly due to higher-order contritbutions to Eq. (7) that modify the relationship between real-space and redshift-space power spectra. For instance, within perturbation theory next-to-leading terms at order $P_{L}^{2}$ arise and have been found to increase the amplitude of the redshift-space power and to improve the agreement with simulations (Taruya et al. 2010; Nishimichi \& Taruya 2011). However, we do not investigate such modifications in this paper.

We do not plot the last " $2 \ell=4$ " term, $\xi_{R_{i}, 4}^{(s)}$, because our analytical computations show that it is very small (smaller than 0.1 on the scales shown in Figs. 1 and 2) and within the error bars. However, we have checked that our model agrees with the simulations for $r>10 h^{-1} \mathrm{Mpc}$ (on smaller scales our model again seems to underestimate this correlation). 
P. Valageas and N. Clerc: Redshift-space correlation functions in large galaxy cluster surveys

\section{Covariance matrices}

\subsection{Explicit expressions}

We now consider the covariance matrices $C_{i, j}^{(s)}$ of the estimators $\hat{\xi}_{R_{i}, 2 \ell_{i}}^{(s)}$, defined by

$C_{i, j}^{(s)}=\left\langle\hat{\xi}_{R_{i}, 2 \ell_{i}}^{(s)} \hat{\xi}_{R_{j}, 2 \ell_{j}}^{(s)}\right\rangle-\left\langle\hat{\xi}_{R_{i}, 2 \ell_{i}}^{(s)}\right\rangle\left\langle\hat{\xi}_{R_{j}, 2 \ell_{j}}^{(s)}\right\rangle$.

To simplify notations, we do not write the labels $R_{i}$ and $2 \ell_{i}$ in the covariance matrices and the subscript $i$ again refers to both the radial bin, $\left[R_{i,-}, R_{i,+}\right]$, and the multipole, $2 \ell_{i}$.

The results of Valageas et al. (2011) directly extend to redshift space and we obtain the expression

$C_{i, j}^{(s)}=C_{i, j}^{(s)(2)}+C_{i, j}^{(s)(3)}+C_{i, j}^{(s)(4)}$,

with (see also Landy \& Szalay 1993; Szapudi 2001; Bernardeau et al. 2002a)

$$
\begin{aligned}
& C_{i, j}^{(s)(2)}=\delta_{R_{i}, R_{j}} \frac{2\left(4 \ell_{i}+1\right)\left(4 \ell_{j}+1\right)}{(\Delta \Omega) Q_{i}^{2}} \int \mathrm{d} \chi_{i} \mathcal{D}_{i}^{2} \frac{\mathrm{d} \boldsymbol{\Omega}_{i}}{(\Delta \Omega)} \frac{\mathrm{d} M_{i}}{M_{i}} \\
& \times \int_{i} \mathrm{~d} s_{i^{\prime}} \frac{\mathrm{d} M_{i^{\prime}}}{M_{i^{\prime}}} \frac{\mathrm{d} n}{\mathrm{~d} \ln M_{i}} \frac{\mathrm{d} n}{\mathrm{~d} \ln M_{i^{\prime}}} \\
& \times P_{2 \ell_{i}}\left(\mu_{i^{\prime}}\right) P_{2 \ell_{j}}\left(\mu_{i^{\prime}}\right)\left[1+\xi_{i, i^{\prime}}^{\mathrm{h}(s)}\right] \text {, } \\
& C_{i, j}^{(s)(3)}=\frac{4\left(4 \ell_{i}+1\right)\left(4 \ell_{j}+1\right)}{(\Delta \Omega) Q_{i} Q_{j}} \int \mathrm{d} \chi_{i} \mathcal{D}_{i}^{2} \frac{\mathrm{d} \boldsymbol{\Omega}_{i}}{(\Delta \Omega)} \frac{\mathrm{d} M_{i}}{M_{i}} \int_{i} \mathrm{~d} s_{i^{\prime}} \frac{\mathrm{d} M_{i^{\prime}}}{M_{i^{\prime}}} \\
& \times \int_{j} \mathrm{~d} s_{j^{\prime}} \frac{\mathrm{d} M_{j^{\prime}}}{M_{j^{\prime}}} \frac{\mathrm{d} n}{\mathrm{~d} \ln M_{i}} \frac{\mathrm{d} n}{\mathrm{~d} \ln M_{i^{\prime}}} \frac{\mathrm{d} n}{\mathrm{~d} \ln M_{j^{\prime}}} \\
& \times P_{2 \ell_{i}}\left(\mu_{i^{\prime}}\right) P_{2 \ell_{j}}\left(\mu_{j^{\prime}}\right)\left[\xi_{i^{\prime}, j^{\prime}}^{\mathrm{h}(s)}+\zeta_{i, i^{\prime}, j^{\prime}}^{\mathrm{h}(s)}\right], \\
& C_{i, j}^{(s)(4)}=\frac{\left(4 \ell_{i}+1\right)\left(4 \ell_{j}+1\right)}{Q_{i} Q_{j}} \int \mathrm{d} \chi_{i} \mathcal{D}_{i}^{2} \frac{\mathrm{d} \boldsymbol{\Omega}_{i}}{(\Delta \Omega)} \frac{\mathrm{d} M_{i}}{M_{i}} \int_{i} \mathrm{~d} s_{i^{\prime}} \frac{\mathrm{d} M_{i^{\prime}}}{M_{i^{\prime}}} \\
& \times \frac{\mathrm{d} n}{\mathrm{~d} \ln M_{i}} \frac{\mathrm{d} n}{\mathrm{~d} \ln M_{i^{\prime}}} \int \mathrm{d} \chi_{j} \mathcal{D}_{j}^{2} \frac{\mathrm{d} \boldsymbol{\Omega}_{j}}{(\Delta \Omega)} \frac{\mathrm{d} M_{j}}{M_{j}} \int_{j} \mathrm{~d} \boldsymbol{s}_{j^{\prime}} \frac{\mathrm{d} M_{j^{\prime}}}{M_{j^{\prime}}} \\
& \times \frac{\mathrm{d} n}{\mathrm{~d} \ln M_{j}} \frac{\mathrm{d} n}{\mathrm{~d} \ln M_{j^{\prime}}} P_{2 \ell_{i}}\left(\mu_{i^{\prime}}\right) P_{2 \ell_{j}}\left(\mu_{j^{\prime}}\right) \\
& \times\left[2 \xi_{i ; j^{\prime}}^{\mathrm{h}(s)} \xi_{i^{\prime} ; j}^{\mathrm{h}(s)}+\eta_{i, i^{\prime} ; j, j^{\prime}}^{\mathrm{h}(s)}\right] .
\end{aligned}
$$

Here we used the same notation as in Valageas et al. (2011) that we recalled below Eq. (22). Thus, labels $i$ and $j$ refer to objects that are at the center of the $\mathcal{V}_{i}$ and $\mathcal{V}_{j}$ shells, whereas labels $i^{\prime}$ and $j^{\prime}$ refer to objects that are within the $\mathcal{V}_{i}$ and $\mathcal{V}_{j}$ shells. The pairs $\left\{i, i^{\prime}\right\}$ and $\left\{j, j^{\prime}\right\}$ are located at unrelated positions in the observational cone, which we integrate over.

In Eq. (30) we note $\delta_{R_{i}, R_{j}}$ the Kronecker symbol with respect to the radial bins. Thus, $\delta_{R_{i}, R_{j}}$ is unity if $\left[R_{i,-}, R_{i,+}\right] \equiv\left[R_{j,-}, R_{j,+}\right]$ and zero otherwise (we consider non-overlapping radial bins), independently of the multipoles $\ell_{i}$ and $\ell_{j}$ (which may be equal or different). This shot-noise contribution arises from cases where the pairs $\left\{i, i^{\prime}\right\}$ and $\left\{j, j^{\prime}\right\}$ are the same (i.e., they involve the same two halos), which implies the same pair length.

The label $C^{(n)}$ refers to quantities that involve $n$ distinct objects. Thus, the contributions $C^{(2)}$ and $C^{(3)}$ arise from shot-noise effects (as is apparent through the prefactors $1 /(\Delta \Omega)$ ), associated with the discreteness of the number density distribution, and they would vanish for continuous distributions. However, they also involve the two-point and three-point correlations, and as such they couple discreteness effects with the underlying largescale correlations of the population. In case of zero large-scale correlations, $C^{(2)}$ remains nonzero because of the unit factor in the brackets and becomes a purely shot-noise contribution, arising solely from discreteness effects. The contribution $C^{(4)}$ is a pure sample-variance contribution and does not depend on the discreteness of the number density distribution (hence there is no $1 /(\Delta \Omega)$ prefactor).

We can reorganize these various terms into three contributions,

$C_{i, j}^{(s)}=C_{i, j}^{(s)(\mathrm{G})}+C_{i, j}^{(s)(\zeta)}+C_{i, j}^{(s)(\eta)}$,

where $C_{i, j}^{(s)(\mathrm{G})}$ gathers all terms that only depend on the two-point correlation, $C_{i, j}^{(s)(\zeta)}$ arises from the three-point correlation (i.e., the last term in Eq. (31)), and $C_{i, j}^{(s)(\eta)}$ arises from the four-point correlation (i.e., the last term in Eq. (32)). We obtain for the Gaussian contribution

$$
\begin{aligned}
C_{i, j}^{(s)(\mathrm{G})}= & \delta_{R_{i}, R_{j}} \frac{2}{(\Delta \Omega) Q_{i}}\left[\delta_{\ell_{i}, \ell_{j}}\left(4 \ell_{i}+1\right)+\left\langle\hat{\xi}_{R_{i} ; 2 \ell_{i}, 2 \ell_{j}}^{(s)}\right\rangle\right] \\
& +\frac{4}{(\Delta \Omega) Q_{i} Q_{j}} \int \mathrm{d} \chi \mathcal{D}^{2} \bar{b}^{2} \bar{n}^{3} \mathcal{V}_{i} \mathcal{V}_{j} \overline{\xi_{i^{\prime}, j^{\prime}}^{(s)}} \\
& +\frac{2}{Q_{i} Q_{j}} \int \mathrm{d} \chi \mathcal{D}^{5} \bar{b} \bar{n}^{4} \mathcal{V}_{i} \mathcal{V}_{j} \overline{\xi_{i ; j^{\prime}}^{(s)} \xi_{i^{\prime} ; j}^{(s)}}
\end{aligned}
$$

which involves terms that are constant, linear, and quadratic over $\xi^{(s)}$. Here we introduced the mean

$\left\langle\hat{\xi}_{R_{i} ; 2 \ell_{i}, 2 \ell_{j}}^{(s)}\right\rangle=\frac{1}{Q_{i}} \int \mathrm{d} \chi \mathcal{D}^{2} \bar{b}^{2} \bar{n}^{2} \mathcal{V}_{i} \overline{\xi_{R_{i} ; 2 \ell_{i}, 2 \ell_{j}}^{(s)}}(z)$,

with

$$
\begin{aligned}
\overline{\xi_{R_{i} ; 2 \ell_{i}, 2 \ell_{j}}^{(s)}}(z)= & \int_{i} \frac{\mathrm{d} s^{\prime}}{\mathcal{V}_{i}}\left(4 \ell_{i}+1\right)\left(4 \ell_{j}+1\right) P_{2 \ell_{i}}\left(\mu^{\prime}\right) P_{2 \ell_{j}}\left(\mu^{\prime}\right) \\
& \times \xi^{(s)}\left(s^{\prime} ; z\right) .
\end{aligned}
$$

From the multipole expansion (11) this also reads as

$$
\begin{aligned}
\overline{\xi_{R_{i} ; 2 \ell_{i}, 2 \ell_{j}}^{(s)}}= & \left(4 \ell_{i}+1\right)\left(4 \ell_{j}+1\right) \int_{R_{i,-}}^{R_{i,+}} \frac{\mathrm{d} r 3 r^{2}}{R_{i,+}^{3}-R_{i,-}^{3}} \\
& \times \sum_{\ell=0}^{2}\left(\begin{array}{ccc}
2 \ell & 2 \ell_{i} & 2 \ell_{j} \\
0 & 0 & 0
\end{array}\right)^{2} \xi^{(s ; 2 \ell)}(r),
\end{aligned}
$$

which involves the square of the Wigner 3-j symbol.

Using the hierarchical ansatz described in Sect. 2.1.3, the contribution $C_{i, j}^{(s)(\zeta)}$ associated with the three-point correlation writes as

$$
\begin{aligned}
C_{i, j}^{(s)(\zeta)}= & \frac{4}{(\Delta \Omega) Q_{i} Q_{j}} \int \mathrm{d} \chi \mathcal{D}^{2} \bar{b}^{3} \bar{n}^{3} \mathcal{V}_{i} \mathcal{V}_{j} \frac{S_{3}}{3} \\
& \times\left[\overline{\xi_{i^{\prime}}^{(s)}} \times \overline{\xi_{j^{\prime}}^{(s)}}+\overline{\xi_{i^{\prime}, i}^{(s)} \xi_{i^{\prime}, j^{\prime}}^{(s)}}+\overline{\xi_{j^{\prime}, \xi^{\prime}}^{(s)} \xi_{j^{\prime}, i^{\prime}}^{(s)}}\right]
\end{aligned}
$$

while the contribution $C_{i, j}^{(s)(\eta)}$ associated with the four-point correlation writes as

$$
\begin{aligned}
C_{i, j}^{(s)(\eta)}= & \frac{2}{Q_{i} Q_{j}} \int \mathrm{d} \chi \mathcal{D}^{5} \bar{b}^{4} \bar{n} \mathcal{V}_{i} \mathcal{V}_{j} \frac{S_{4}}{16}\left[\overline{\xi_{i^{\prime}}^{(s)}} \times \overline{\xi_{i ; j} \xi_{i ; j^{\prime}}^{(s)}}\right. \\
& +\overline{\xi_{j^{\prime}}^{(s)}} \times \overline{\xi_{i ; j} \xi_{j ; i^{\prime}}^{(s)}}+2 \overline{\xi_{i^{\prime}}^{(s)}} \times \overline{\xi_{j^{\prime}}^{(s)}} \times \overline{\xi_{\mathrm{cyl}}} \\
& \left.+2 \overline{\xi_{j^{\prime} ;}^{(s)} \xi_{i ; j} \xi_{j ; i^{\prime}}^{(s)}}+\overline{\xi_{j^{\prime} ; i}^{(s)} \xi_{i, i^{\prime}}^{(s)} \xi_{i^{\prime} ; j}^{(s)}}+\overline{\xi_{i^{\prime} ; j}^{(s)} \xi_{j, j^{\prime}}^{(s)} \xi_{j^{\prime} ; i}^{(s)}}\right]
\end{aligned}
$$




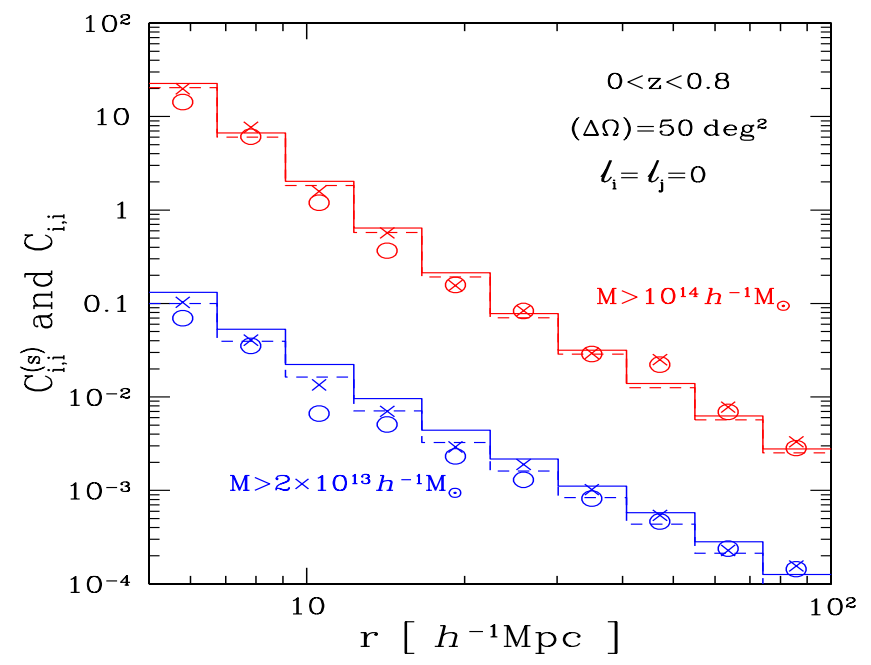

Fig. 3. Redshift-space (solid line, for $2 \ell=0$ ) and real-space (dashed line) covariance matrices, $C_{i, j}^{(s)}$ and $C_{i, j}$, along the diagonal, $i=j$. We show the results obtained for halos in the redshift range $0<z<0.8$ with an angular window of $50 \mathrm{deg}^{2}$.

Here the quantities that are overlined are various geometrical averages of the redshift-space two-point correlation functions. For instance, the geometrical average introduced in the first integral in Eq. (34) writes as

$$
\begin{aligned}
\overline{\xi_{i^{\prime}, j^{\prime}}^{(s)}}(z)= & \left(4 \ell_{i}+1\right)\left(4 \ell_{j}+1\right) \int_{i} \frac{\mathrm{d} s_{i^{\prime}}}{\mathcal{V}_{i}} P_{2 \ell_{i}}\left(\mu_{i^{\prime}}\right) \int_{j} \frac{\mathrm{d} s_{j^{\prime}}}{V_{j}} P_{2 \ell_{j}}\left(\mu_{j^{\prime}}\right) \\
& \times \xi^{(s)}\left(s_{i^{\prime}}-s_{j^{\prime}} ; z\right) .
\end{aligned}
$$

We give the explicit expressions of these various terms in Appendix A, and we describe how angular integrations can be performed using the spherical harmonic decomposition (11), as well as the flat-sky and Limber's approximations. All terms involve the redshift-space correlation $\xi^{(s)}$, except for $\overline{\xi_{\text {cyl }}}$, which only depends on the power along the transverse directions (and is not affected by redshift-space distortions).

\subsection{Covariance matrix of the monopole redshift-space correlation}

In this section we study the covariance matrix $C_{i, j}^{(s)}$ of the monopole redshift-space correlation, $\xi_{R_{i}, 0}^{(s)}$, that is, $\ell_{i}=\ell_{j}=0$.

\subsubsection{Comparison between redshift-space and real-space covariance matrices}

We show in Figs. 3 and 4 the redshift-space and real-space covariance matrices $C_{i, j}^{(s)}$ and $C_{i, j}$, for halos in the redshift range $0<z<0.8$. The redshift-space covariance matrix is greater than the real-space covariance matrix by about $11 \%$ along the diagonal and by $20 \%$ for off-diagonal entries, for massive halos, $M>10^{14} h^{-1} M_{\odot}$. For $M>2 \times 10^{13} h^{-1} M_{\odot}$, these amplification factors are higher, $33 \%$ along the diagonal and $40 \%$ for off-diagonal entries. This could be expected from the higher value of the redshift-space correlation function noticed in Fig. 1. This is due to the amplification of the redshift-space power spectrum through the Kaiser effect, as shown by Eq. (9). Again, the relative effect is weaker for rare massive halos because of their larger bias $\bar{b}$, which decreases the associated factor $\beta=f / \bar{b}$.

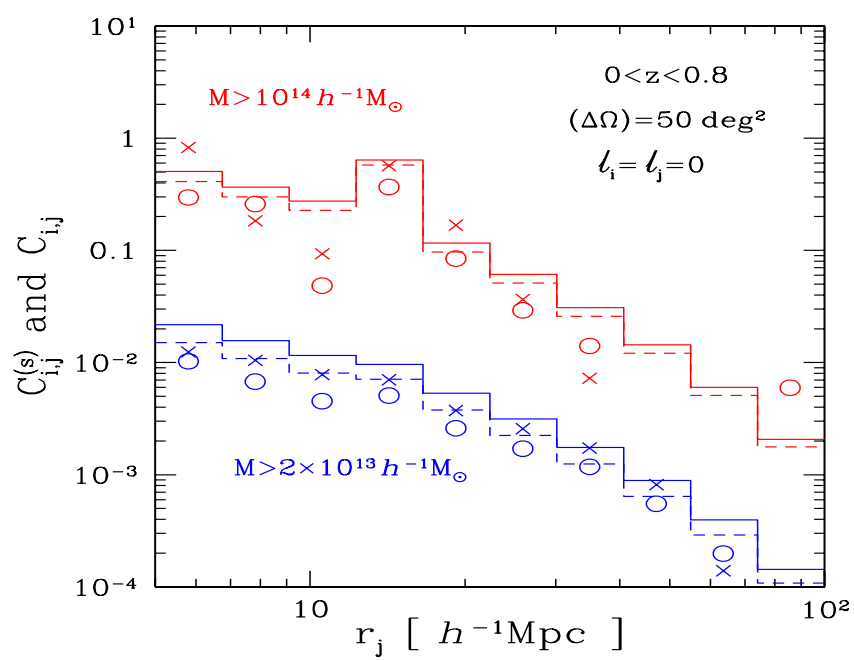

Fig. 4. Redshift-space (solid line, for $2 \ell=0$ ) and real-space (dashed line) covariance matrices, $C_{i, j}^{(s)}$ and $C_{i, j}$, as a function of $j$, for $i=4$ associated with the distance bin $12.3<r<16.6 h^{-1}$ Mpc. We show the results obtained for halos in the redshift range $0<z<0.8$ with an angular window of $50 \mathrm{deg}^{2}$.

The amplification of the covariance matrix is lower along the diagonal, where the covariance matrix includes a pure shot-noise term (first term in Eq. (34)) that is independent of $\xi^{(s)}$ and is not amplified by the Kaiser effect.

We obtain a reasonably good agreement with the numerical simulations for both redshift-space and real-space covariance matrices, for diagonal and off-diagonal entries. The simulation data are somewhat noisier for off diagonal entries and show spurious oscillations in Fig. 4 that are not physical but due to low statistics, especially for rare massive halos. This shows that analytic models are competitive tools to estimate these covariance matrices. In particular, for data analysis purposes it can be helpful to have smooth covariance matrices, which make matrix inversion more reliable.

In any case, redshift-space distortions only have a moderate impact on the covariance matrices and do not change their magnitude. This means that most of the real-space results obtained in Valageas et al. (2011) remain valid.

We show in Fig. 5 an estimate of the signal-to-noise ratio of the redshift-space and real-space two-point correlations, defined as $\left\langle\hat{\xi}_{R_{i}}\right\rangle / \sqrt{C_{i, i}}$. A more precise analysis would require using the full covariance matrix, but this ratio should allow us to check whether redshift distortions, which amplify both the mean correlation and its covariance matrix, degrade the signal-to-noise ratio. Clearly, Fig. 5 shows that the signal-to-noise ratio is not strongly affected by the Kaiser effect. Indeed, the amplification of $\hat{\xi}_{R_{i}, 0}^{(s)}$ overweights the amplification of $C_{i, j}^{(s)}$ and the signal-tonoise ratio slightly increases. This can be understood because the contributions to $C_{i, j}^{(s)}$ that are cubic over $\xi^{(s)}$ (or of higher order), which in our framework only come from the four-point correlation contribution (39) to the pure sample-variance term, are less important than the mixed shot-noise and sample-variance contributions that are constant or linear over $\xi^{(s)}$.

The results found in Fig. 5 mean that redshift distortions do not prevent measuring the clustering of X-ray clusters and its use to constrain cosmology, since the signal-to-noise ratio remains as high and even slightly higher. 
P. Valageas and N. Clerc: Redshift-space correlation functions in large galaxy cluster surveys

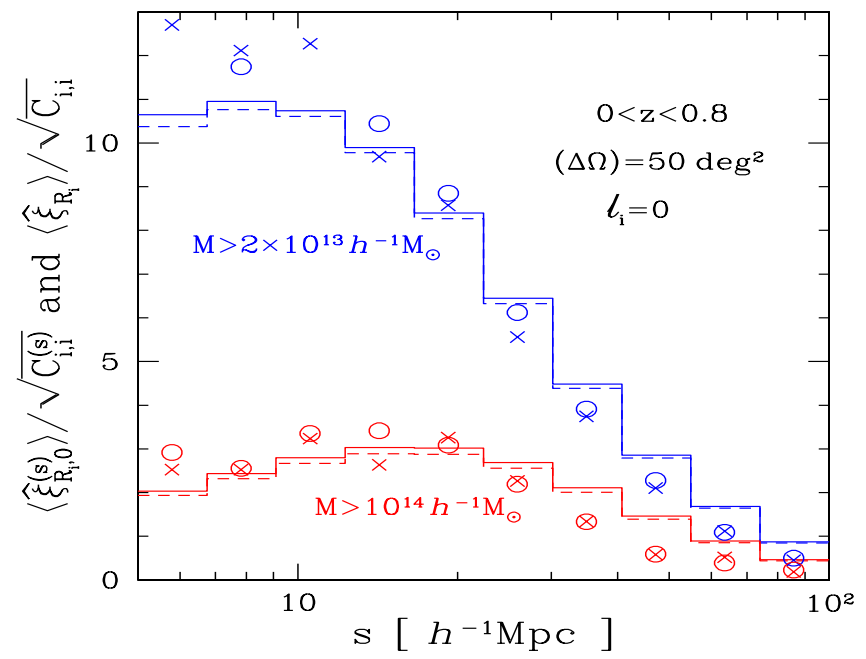

Fig. 5. Signal-to-noise ratio of the redshift-space (solid line) and realspace (dashed line) two-point correlations. We show the results obtained for halos in the redshift range $0<z<0.8$ with an angular window of $50 \mathrm{deg}^{2}$

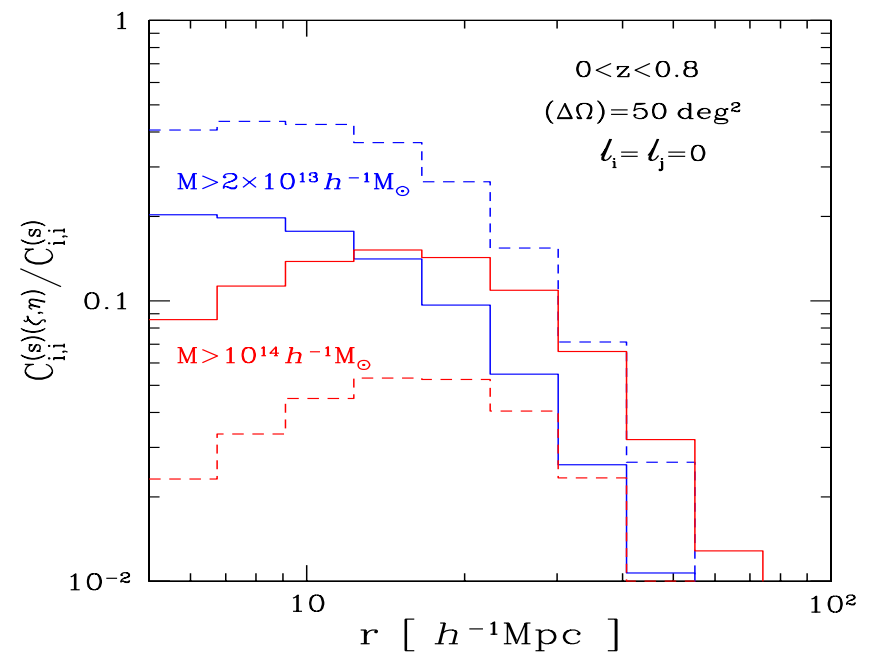

Fig. 6. Relative importance of the non-Gaussian contributions $C_{i, j}^{(s)(\zeta)}$ (solid line) and $C_{i, j}^{(s)(\eta)}$ (dashed line) to the redshift-space covariance matrix $C_{i, j}^{(s)}$, along the diagonal, $i=j$. We show the results obtained for halos in the redshift range $0<z<0.8$ with an angular window of $50 \mathrm{deg}^{2}$.

\subsubsection{Importance of non-Gaussian contributions}

We show in Figs. 6 and 7 the ratios $C_{i, j}^{(s)(\zeta)} / C_{i, j}^{(s)}$ and $C_{i, j}^{(s)(\eta)} / C_{i, j}^{(s)}$. They give the relative contribution to the covariance matrix of the terms associated with the three-point correlation function, Eq. (38), or with the four-point correlation function, Eq. (39). For low-mass halos the 4-pt contribution $C_{i, j}^{(s)(\eta)}$ is larger than the 3-pt contribution $C_{i, j}^{(s)(\zeta)}$, whereas the ordering is reversed for high-mass halos. This agrees with the results obtained in realspace in Valageas et al. (2011) (see their Figs. 15 and 16). This behavior is due to the greater importance of shot-noise effects for rare massive halos. This gives more weight to the 3-pt contribution, which is part of the coupled shot-noise-sample-variance contribution $C^{(s)(3)}$ in Eq. (31), than to the 4-pt contribution, which is part of the pure sample-variance contribution $C^{(s)(4)}$ in Eq. (32). For the same reason, these non-Gaussian contributions

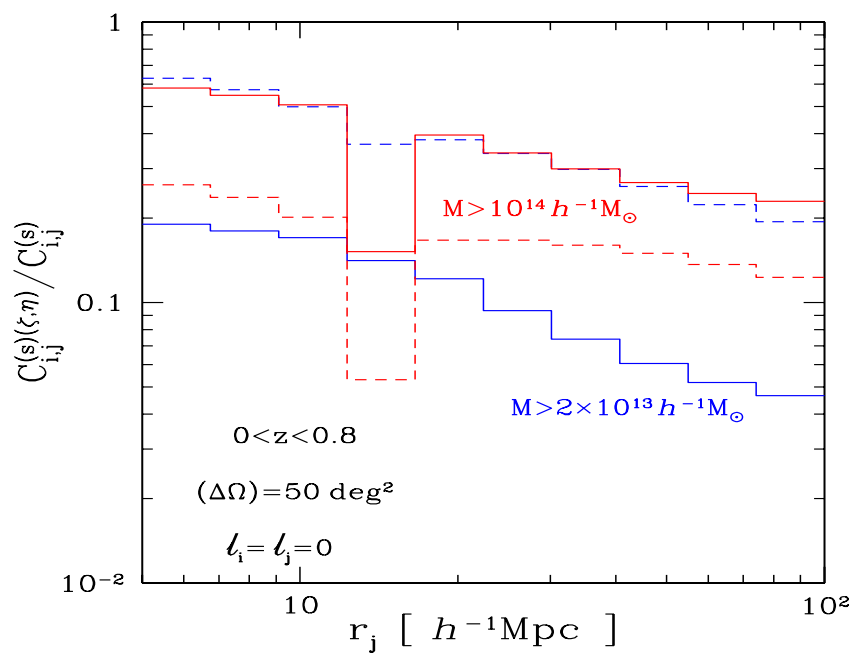

Fig. 7. Relative importance of the non-Gaussian contributions $C_{i, j}^{(s)(\zeta)}$ (solid line) and $C_{i, j}^{(s)(\eta)}$ (dashed line) to the redshift-space covariance matrix $C_{i, j}^{(s)}$, along one row $(i=4)$, associated with the distance bin $12.3<r<16.6 h^{-1} \mathrm{Mpc}$. We show the results obtained for halos in the redshift range $0<z<0.8$ with an angular window of $50 \mathrm{deg}^{2}$.

are relatively smaller along the diagonal for rare halos, as seen on the fourth bin $j=i$ in Fig. 7 .

Figures 6 and 7 show that the non-Gaussian contributions can make up to $20-60 \%$ of the full covariance matrix as soon as one bin $i$ or $j$ corresponds to scales on the order of $10 h^{-1} \mathrm{Mpc}$. Their relative contribution is also somewhat larger for offdiagonal terms. Therefore, although these terms do not change the order of magnitude of the covariance matrix, it is necessary to include them to obtain accurate estimates.

\subsubsection{Correlation matrices}

We show in Fig. 8 the redshift-space correlation matrices defined as

$\mathcal{R}_{i, j}^{(s)}=\frac{C_{i, j}^{(s)}}{\sqrt{C_{i, i}^{(s)} C_{j, j}^{(s)}}}$.

We plot the correlation matrices associated with the Gaussian part (34) and with the full matrix (33). In agreement with Fig. 7, we can see that the non-Gaussian contributions, associated with the 3-pt and 4-pt correlations, make the correlation matrix less diagonal, especially for low-mass halos. As explained above, this is because rare massive halos show stronger pure shot-noise effects that give an additional weight to the diagonal entries. In any case, we can see that it is important to take into account nonGaussian contributions to obtain accurate estimates. Moreover, off-diagonal terms cannot be neglected (especially for low-mass halos), hence it is necessary to use the full covariance matrix $C_{i, j}^{(s)}$ to draw constraints on cosmology from the analysis of X-ray cluster surveys.

\subsection{Covariance matrix of the " $2 \ell=2$ " redshift-space correlation}

We now study the covariance matrix $C_{i, j}^{(s)}$ of the " $2 \ell=2$ " redshift-space correlation, $\xi_{R_{i}, 2}^{(s)}$, that is, $2 \ell_{i}=2 \ell_{j}=2$. 

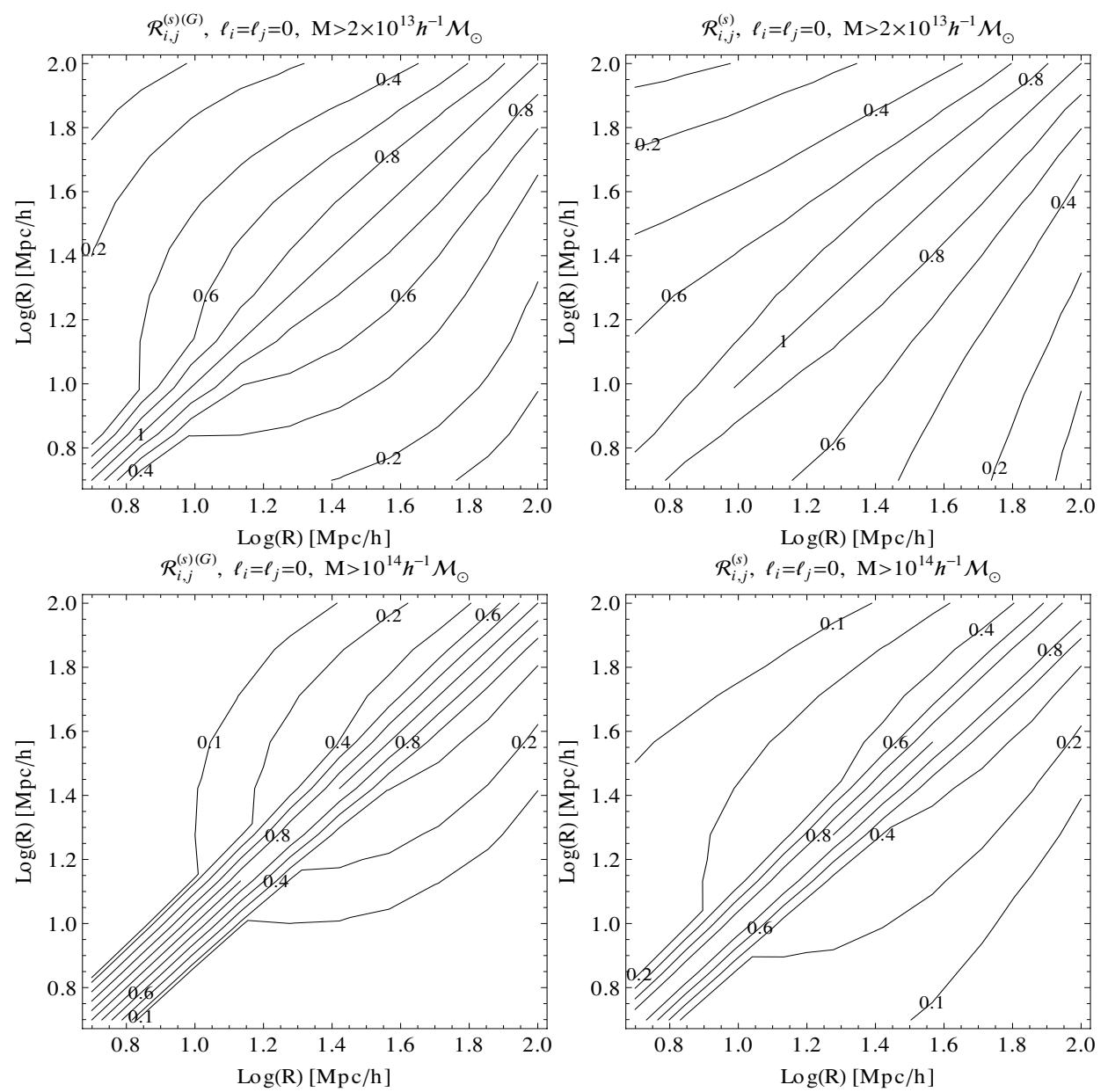

Fig. 8. Contour plots for the redshift-space correlation matrices $\mathcal{R}_{i, j}^{(s)}$, defined as in Eq. (41). There are ten distance bins, over $5<r<100 h^{-1} \mathrm{Mpc}$, equally spaced in $\log (r)$, as in previous figures. We considered halos in the redshift range $0<z<0.8$, within an angular window of 50 deg ${ }^{2}$, above the mass thresholds $M>2 \times 10^{13} h^{-1} M_{\odot}$ in the upper row, and $M>10^{14} h^{-1} M_{\odot}$ in the lower row. Left panels: correlation matrix $\mathcal{R}_{i, j}^{(s)(G)}$ associated with the Gaussian part (34) of the covariance matrix. Right panels: full correlation matrix, associated with the full matrix (33).

We compare in Figs. 9 and 10 our analytical model with numerical simulations for the covariance matrix along the diagonal and along one row. We considered two analyses of the numerical simulations, where we used either 138 nonoverlapping fields separated by a 10 degree gap (squares) or 34 fields separated by a 20 degree gap (crosses). This gives a measure of the statistical error of the simulation data, apart from the systematic finite-resolution effects. Along the diagonal (Fig. 9) our predictions show reasonable agreement with the simulations, which appear reliable with low noise. Along one row (Fig. 10) the simulations give higher off-diagonal entries than our model, but they also show a significant statistical noise. In view of these uncertainties we obtain a reasonable agreement on scales above $10 h^{-1} \mathrm{Mpc}$. On smaller scales, our model may underestimate the off-diagonal covariance because of nonlinear effects, as suggested by the comparison for the mean correlation itself shown in Fig. 2. Therefore, on scales above $10 \mathrm{~h}^{-1} \mathrm{Mpc}$ analytical models such as the one presented in this paper can provide a competitive alternative to numerical simulations to estimate quantities such as covariance matrices that are difficult to obtain from numerical simulations (as they require large box sizes with a good resolution and time-consuming data analysis procedures).

We show in Figs. 11 and 12 the relative contribution to the covariance matrix of the terms associated with the 3-pt and 4-pt correlation functions. The comparison with Figs. 6 and 7 shows that these non-Gaussian contributions are relatively less important along the diagonal but more important on off-diagonal entries than for the monopole " $2 \ell=0$ " case. Thus, they make up to $40-60 \%$ of the full covariance matrix over a large range of scales.

For completeness we show in Appendix $\mathrm{C}$ the correlation matrices of this " $2 \ell=2$ " redshift-space correlation, as well as the cross-correlation between the " $2 \ell=0$ " and " $2 \ell=2$ " components. We find that the " $2 \ell=2$ " covariance matrix is significantly more diagonal than the " $2 \ell=0$ " covariance matrix and that the cross-correlation matrix between both components is quite small $(\sim 0.05)$. This means that the full covariance matrix in the $\left\{R_{i}, \ell_{i}\right\}$ space is approximately block-diagonal (we may neglect entries with $\ell_{i} \neq \ell_{j}$ ). Then, we can decouple the analysis of $\xi_{R_{i}, 0}^{(s)}$ and $\xi_{R_{i}, 2}^{(s)}$.

\section{Applications to real survey cases}

As in Valageas et al. (2011), we now study the correlation functions that can be measured in several cluster surveys. We considered three surveys on limited angular windows:

- The XXL survey (Pierre et al. 2011) is an XMM Very Large Programme specifically designed to constrain the equation of state of the dark energy by using clusters of galaxies. It consists of two $25 \mathrm{deg}^{2}$ areas and probes massive clusters out to 
P. Valageas and N. Clerc: Redshift-space correlation functions in large galaxy cluster surveys

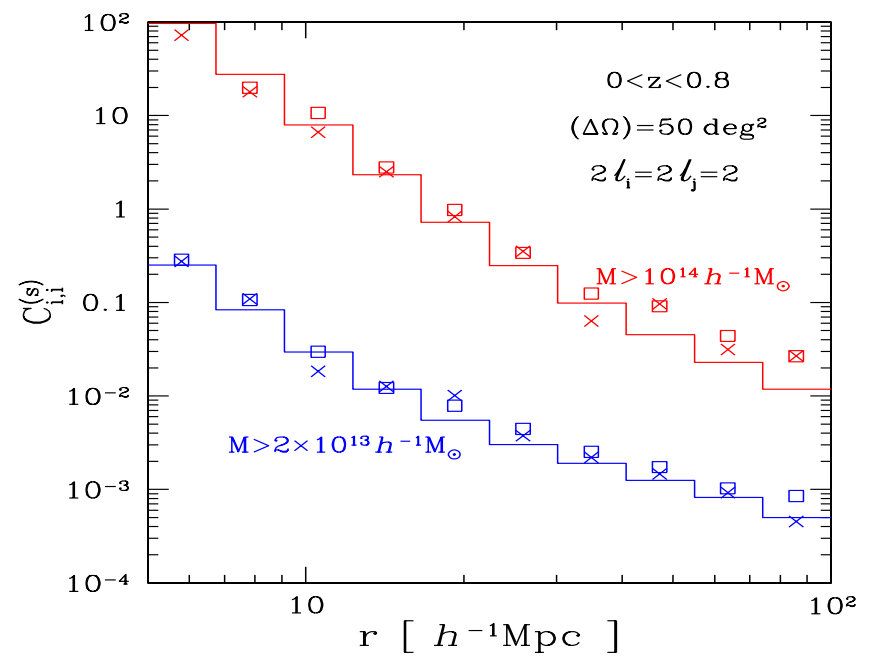

Fig. 9. Covariance matrix $C_{i, j}^{(s)}$ along the diagonal, $i=j$, for the " $2 \ell=2$ " mutipole of the redshift-space correlation. We show the results obtained for halos in the redshift range $0<z<0.8$ with an angular window of $50 \mathrm{deg}^{2}$. Points from numerical simulations are obtained from 138 fields separated by a 10 degree gap (squares) or from 34 fields separated by 20 degree gap (crosses).

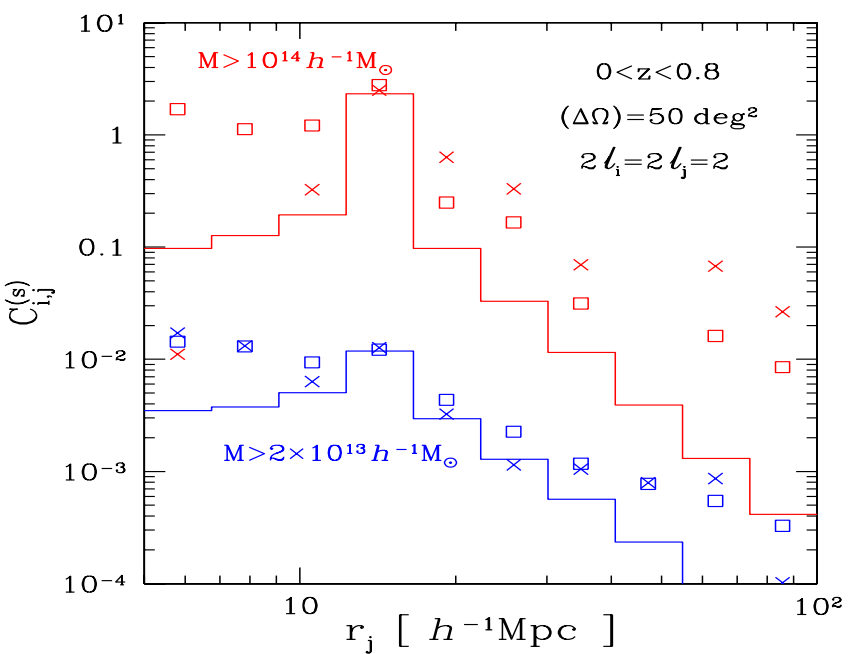

Fig. 10. Covariance matrix $C_{i, j}^{(s)}$ as a function of $j$, for $i=4$ associated with the distance bin $12.3<r<16.6 h^{-1} \mathrm{Mpc}$, for the " $2 \ell=2$ " mutipole of the redshift-space correlation. We show the results obtained for halos in the redshift range $0<z<0.8$ with an angular window of $50 \mathrm{deg}^{2}$.

a redshift of $\sim 2$. We considered the "C1 selection function" given in Fig. J.1 of Valageas et al. (2011, see also Pacaud et al. 2006, 2007).

- The Dark Energy Survey (DES) is an optical imaging survey to cover $5000 \mathrm{deg}^{2}$ with the Blanco four-meter telescope at the Cerro Tololo Inter-American Observatory ${ }^{2}$. We considered the expected mass threshold $M>5 \times$ $10^{13} h^{-1} M_{\odot}$, as well as the subset of massive clusters $M>$ $5 \times 10^{14} h^{-1} M_{\odot}$.

- The South Pole Telescope (SPT) operates at millimeter wavelengths ${ }^{3}$. It will cover some $2500 \mathrm{deg}^{2}$ at three frequencies, aiming at detecting clusters of galaxies from the

\footnotetext{
${ }^{2}$ https://www . darkenergysurvey.org/index.shtml

${ }^{3}$ http://pole.uchicago.edu/
}

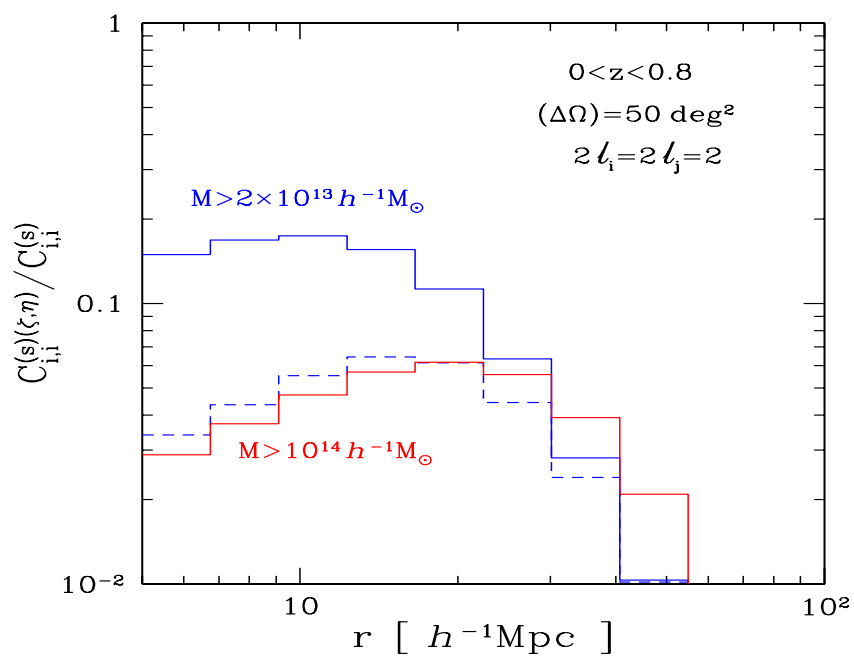

Fig. 11. Relative importance of the non-Gaussian contributions $C_{i, j}^{(s)(\zeta)}$ (solid line) and $C_{i, j}^{(s)(\eta)}$ (dashed line) to the redshift-space covariance matrix $C_{i, j}^{(s)}$, along the diagonal, $i=j$. We show the results obtained for halos in the redshift range $0<z<0.8$ with an angular window of $50 \mathrm{deg}^{2}$. (The contribution $C_{i, j}^{(s)(\eta)}$ for $M>10^{14} h^{-1} M_{\odot}$ does not appear because it is less than $10^{-2}$.)

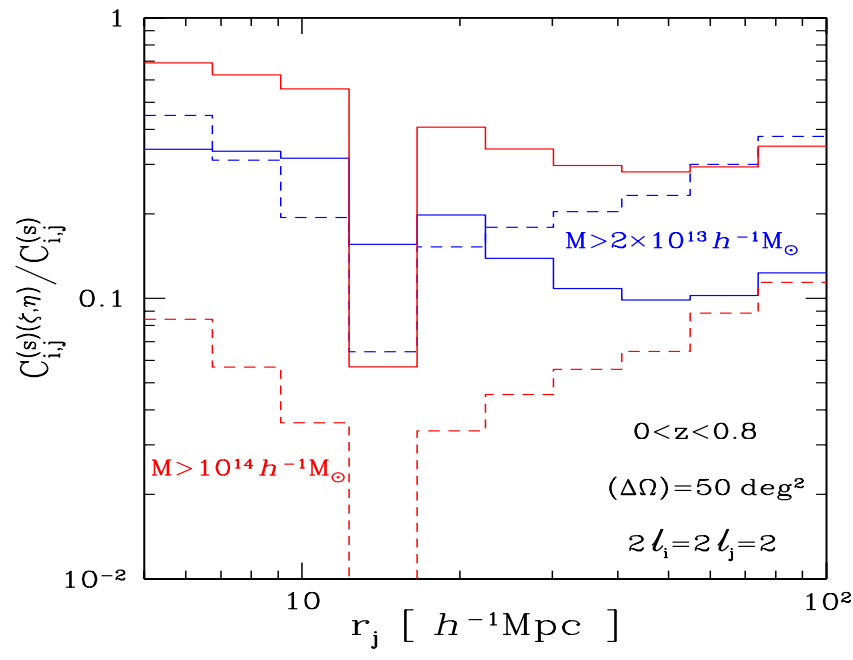

Fig. 12. Relative importance of the non-Gaussian contributions $C_{i, j}^{(s)(\zeta)}$ (solid line) and $C_{i, j}^{(s)(\eta)}$ (dashed line) to the redshift-space covariance matrix $C_{i, j}^{(s)}$, along one row $(i=4)$, associated with the distance bin $12.3<r<16.6 h^{-1} \mathrm{Mpc}$. We show the results obtained for halos in the redshift range $0<z<0.8$ with an angular window of $50 \mathrm{deg}^{2}$.

Sunyaev-Zel'dovich (S-Z) effect. We considered a mass threshold of $5 \times 10^{14} h^{-1} M_{\odot}$ (Vanderlinde et al. 2010).

We also considered three all-sky surveys. In practice, the total angular area of these surveys is not really $4 \pi$ sterad since we must remove the galactic plane. In the following, for Planck we considered the two-sided cone of angle $\theta_{\mathrm{s}}=75 \mathrm{deg}$ (i.e., $|b|>15 \mathrm{deg}$ ), which yields a total area $\Delta \Omega \simeq 30576 \mathrm{deg}^{2}$. For Erosita and Euclid we took $\theta_{\mathrm{s}}=59 \mathrm{deg}$ (i.e., $|b|>31 \mathrm{deg}$ ), which corresponds to a total area that is about one-half of the full sky, $\Delta \Omega \simeq 20000 \mathrm{deg}^{2}$.

- Planck operates at nine frequencies, enabling an efficient detection of the cluster $\mathrm{S}-\mathrm{Z}$ signature but has a rather large PSF $\left(5^{\prime}-10^{\prime}\right)$. Some 1625 massive clusters out to $z=1$ are expected over the whole sky. We assumed the selection 


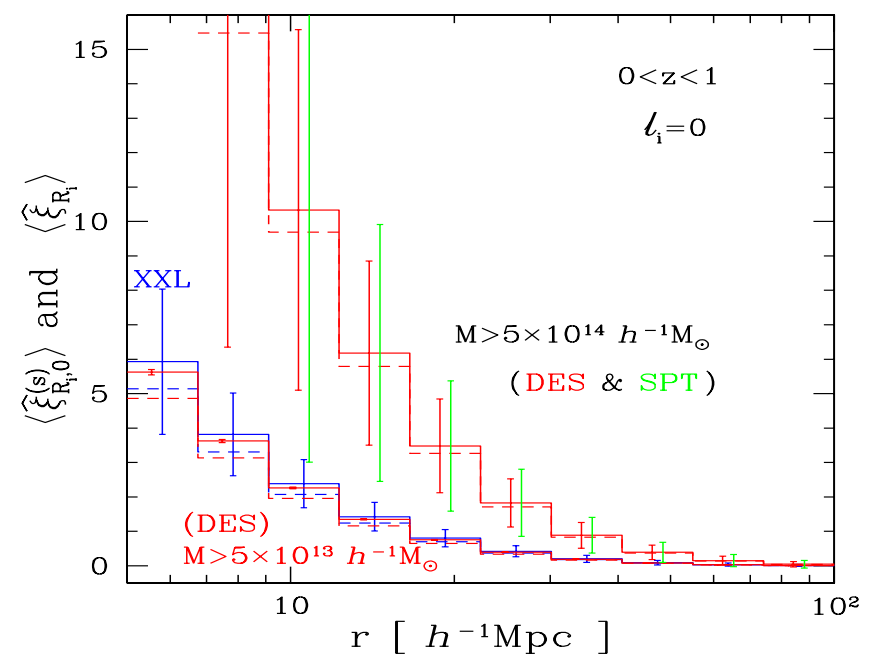

Fig. 13. Mean monopole redshift-space (solid lines) and real-space (dashed lines) correlations, $\left\langle\hat{\xi}_{R_{i}, 0}^{(s)}\right\rangle$ and $\left\langle\hat{\xi}_{R_{i}}\right\rangle$, over ten comoving distance bins within $5<r<100 h^{-1} \mathrm{Mpc}$, equally spaced in $\log (r)$. We integrated over halos within the redshift interval $0<z<1$, for the XXL, DES, and SPT surveys. For DES we considered the mass thresholds $M>5 \times 10^{13} h^{-1} M_{\odot}$ and $M>5 \times 10^{14} h^{-1} M_{\odot}$ (smaller error bars), and for SPT the mass threshold $M>5 \times 10^{14} h^{-1} M_{\odot}$ (larger error bars shifted to the right). The error bars show the diagonal part of the redshift-space covariance $\sigma_{\xi_{i}^{(s)}}=\sqrt{C_{i, i}^{(s)}}$.

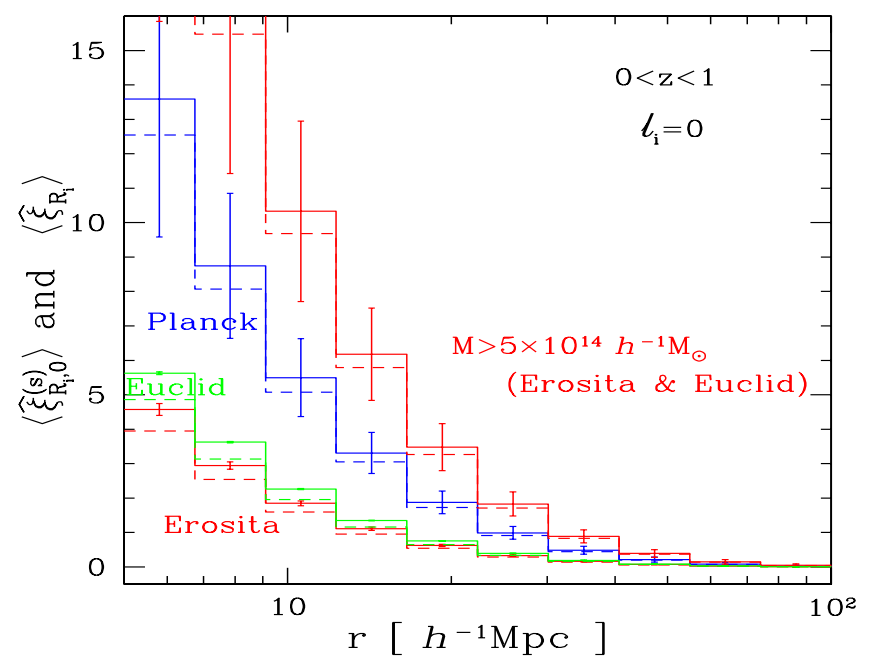

Fig. 14. Mean monopole correlations, $\left\langle\hat{\xi}_{R_{i}, 0}^{(s)}\right\rangle$ (solid lines) and $\left\langle\hat{\xi}_{R_{i}}\right\rangle$ (dashed lines), as in Fig. 13 but for all-sky surveys. From top to bottom, we show a) halos above $5 \times 10^{14} h^{-1} M_{\odot}$ in either Erosita or Euclid, b) halos detected by Planck, c) halos above $5 \times 10^{13} h^{-1} M_{\odot}$ in Euclid, and d) halos detected by Erosita.

function by Melin et al. (2006), shown in Fig. J.1 of Valageas et al. (2011).

- For Erosita, a simple flux limit is currently assumed as an average over the whole sky: $4 \times 10^{-14} \mathrm{erg} \mathrm{s}^{-1} \mathrm{~cm}^{-2}$ in the [0.5-2] keV band (Predehl et al. 2009). The associated selection function is shown in Fig. J.1 of Valageas et al. (2011). This would yield 71907 clusters out to $z=1$.

- For Euclid, we followed the prescription of the Euclid science book for the cluster optical selection function and adopted a fixed mass threshold of $5 \times 10^{13} h^{-1} M_{\odot}$ (Refregier et al. 2011).

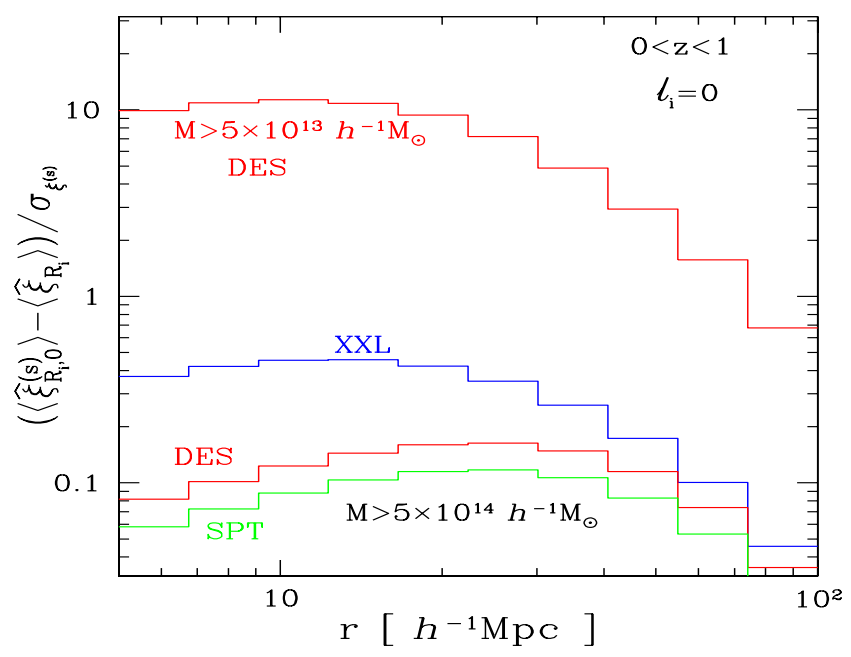

Fig. 15. Ratio $\left(\left\langle\hat{\xi}_{R_{i}, 0}^{(s)}\right\rangle-\left\langle\hat{\xi}_{R_{i}}\right\rangle\right) / \sigma_{\xi_{i}^{(s)}}$, for the surveys shown in Fig. 13.

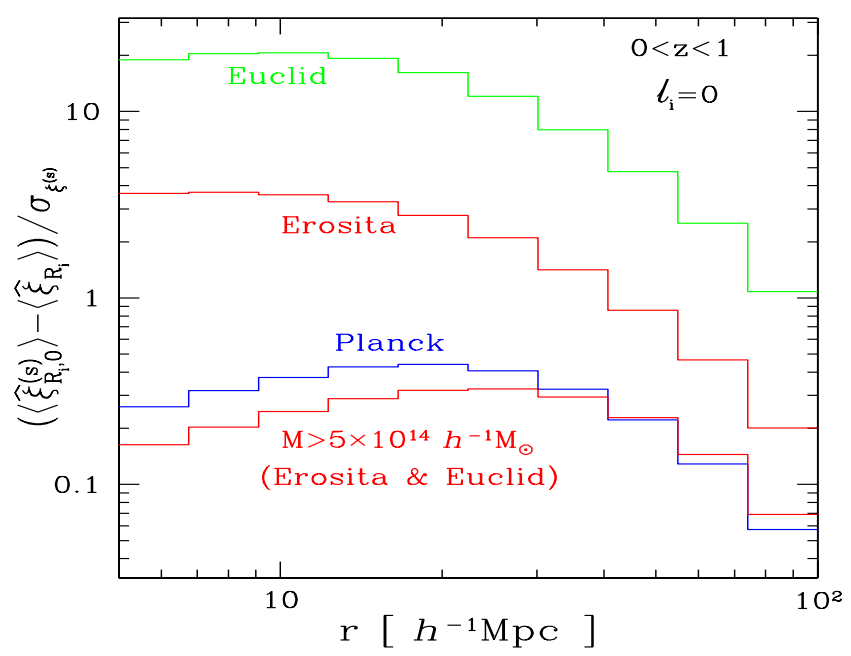

Fig. 16. Ratio $\left(\left\langle\hat{\xi}_{R_{i}, 0}^{(s)}\right\rangle-\left\langle\hat{\xi}_{R_{i}}\right\rangle\right) / \sigma_{\xi_{i}^{(s)}}$, for the surveys shown in Fig. 14 .

In the following, the error bars shown in the figures are the statistical error bars associated with the covariance matrices studied in Sect. 4 (i.e., we did not include other sources of uncertainties such as observational noise). For the full-sky surveys this "sample-variance" noise does not vanish because even full-sky surveys up to a given redshift only cover a finite volume. In this limit the "sample variance" is usually called the "cosmic variance".

\subsection{Monopole redshift-space correlation}

We show the redshift-space and real-space correlations $\left\langle\hat{\xi}_{R_{i}, 0}^{(s)}\right\rangle$ and $\left\langle\hat{\xi}_{i}\right\rangle$ that can be measured with these surveys in the redshift range $0<z<1$ in Figs. 13 and 14. In each case, the redshiftspace correlation is slightly higher than the real-space correlation. The error bars $\sigma_{\xi_{i}^{(s)}}=\sqrt{C_{i, i}^{(s)}}$ correspond to the diagonal entries of the redshift-space covariance matrix.

Redshift-space distortions can be seen in these figures, but in several cases they are smaller than the error bars. To see the impact of these redshift-space distortions more clearly, we show the ratio $\left(\left\langle\hat{\xi}_{R_{i}, 0}^{(s)}\right\rangle-\left\langle\hat{\xi}_{R_{i}}\right\rangle\right) / \sigma_{\xi_{i}^{(s)}}$ in Figs. 15 and 16. This gives the difference between the monopole redshift-space and real-space correlations in units of the standard deviation $\sigma_{\xi_{i}^{(s)}}$, that is, of the error bar of the measure. 
P. Valageas and N. Clerc: Redshift-space correlation functions in large galaxy cluster surveys

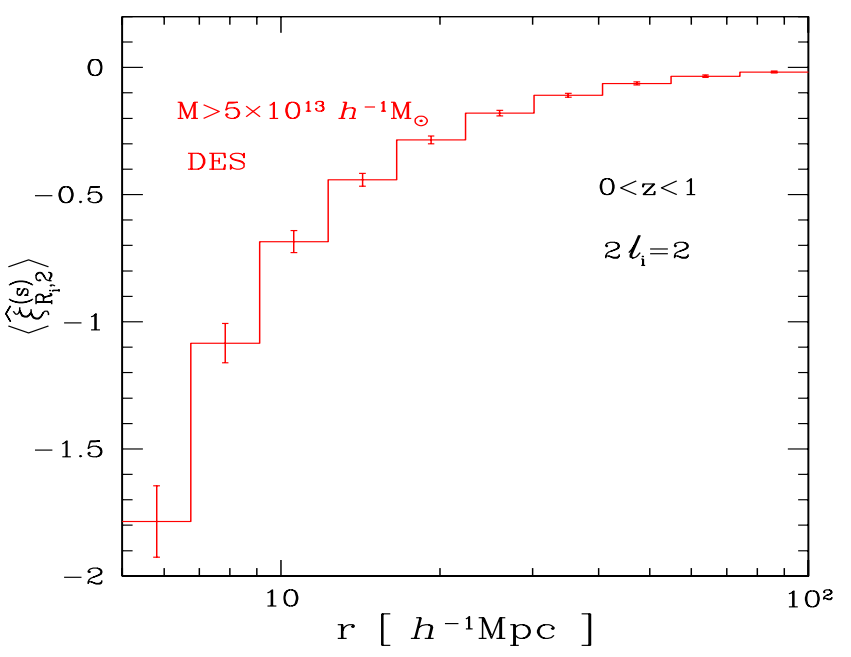

Fig. 17. Mean " $2 \ell=2$ " redshift-space correlation $\left\langle\hat{\xi}_{R_{i}, 2}^{(s)}\right\rangle$. As in Fig. 13, we considered ten comoving distance bins within $5<r<100 h^{-1} \mathrm{Mpc}$, equally spaced in $\log (r)$, and integrated over halos within the redshift interval $0<z<1$. We show our results for halos above $M>5 \times$ $10^{13} h^{-1} M_{\odot}$ for DES. The error bars show the diagonal part of the redshift-space covariance $\sigma_{\xi_{i}^{(s)}}=\sqrt{C_{i, i}^{(s)}}$.

Among limited-area surveys, the amplification of the cluster correlation function by the Kaiser effect will be clearly seen for halos above $M>5 \times 10^{13} h^{-1} M_{\odot}$ in DES. The effect is negligible for more massive halos, $M>5 \times 10^{14} h^{-1} M_{\odot}$, in DES and $\mathrm{SPT}$, because of their larger error bars (see Fig. 13). For the " $\mathrm{C} 1$ clusters" measured in the XXL survey redshift distortions are marginally relevant: they are on the order of $0.4 \sigma_{\xi_{i}^{(s)}}$ in distance bins around $15 h^{-1} \mathrm{Mpc}$, so that by collecting the signal from all distance bins they would give a signal-to-noise ratio of about unity.

Among full-sky surveys, the redshift-space amplification will have a clear impact for the full cluster populations measured in Erosita and Euclid, and a marginal impact for Planck. Again, subsamples of massive clusters, $M>5 \times 10^{14} h^{-1} M_{\odot}$, lead to large error bars that hide this redshift-space effect.

In practice one does not measure both real-space and redshift-space correlations from a cluster survey, because one does not have a map of the cluster velocities. Therefore, the redshift-space amplification of the spherically averaged correlation $\left\langle\hat{\xi}_{R_{i}, 0}^{(s)}\right\rangle$ is degenerate with the halo bias $\bar{b}$ (which is difficult to predict with a high accuracy). To measure redshift-space distortions, one needs to measure the anisotropies of the redshift-space correlation, that is, the higher-order multipoles $\xi^{(s ; 2)}$ and $\xi^{(s ; 4)}$ of Eq. (11). This provides a distinctive signature that breaks the degeneracy with the halo bias and yields another constraint on cosmology through the factor $\beta$, that is, the growth rate $f$ of the density fluctuations defined in Eq. (8). We consider these higherorder multipoles in the following sections.

\section{2. " $2 \ell=2$ " redshift-space correlation}

We show in Figs. 17 and 18 the mean " $2 \ell=2$ " redshiftspace correlation $\left\langle\hat{\xi}_{R_{i}, 2}^{(s)}\right\rangle$. We considered the same surveys as in Sect. 5.1, but we only plot our results for the cases where the signal-to-noise ratio is higher than unity. In agreement with the analysis of Sect. 3, this quadrupole term is more difficult to measure than the monopole term studied in Sect. 5.1, and only DES, Erosita, and Euclid can obtain a clear detection. Even for

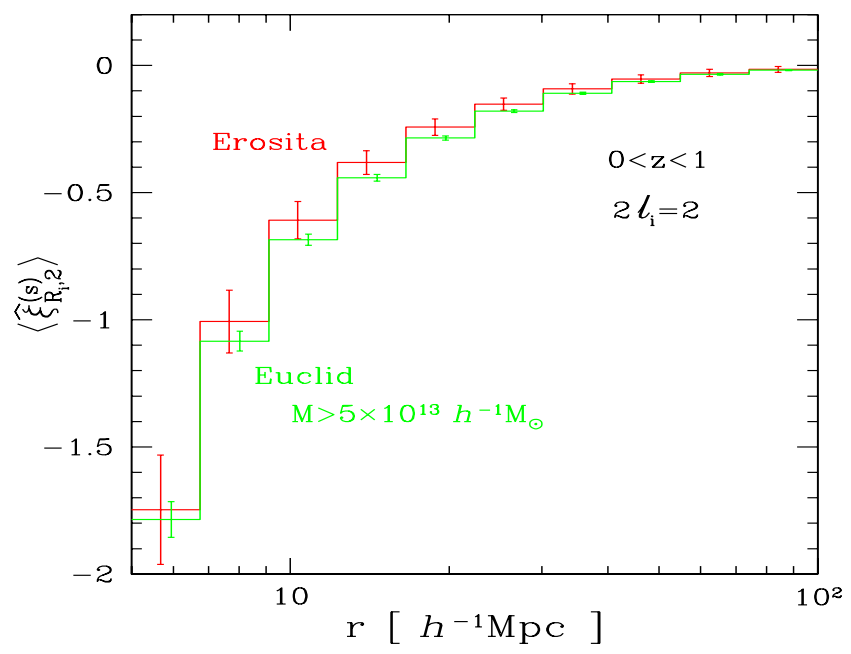

Fig. 18. Mean " $2 \ell=2$ " redshift-space correlation $\left\langle\hat{\xi}_{R_{i}, 2}^{(s)}\right\rangle$, as in Fig. 17 but for all-sky surveys. We show our results for all halos detected by Erosita (upper curve) and for halos above $5 \times 10^{13} h^{-1} M_{\odot}$ in Euclid (lower curve).

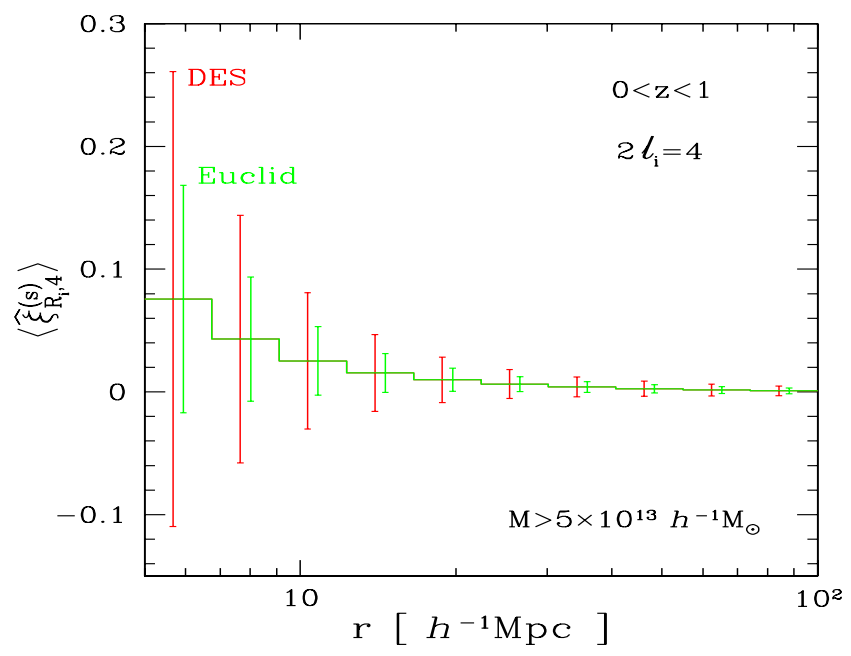

Fig. 19. Mean " $2 \ell=4$ "redshift-space correlation $\left\langle\hat{\xi}_{R_{i}, 4}^{(s)}\right\rangle$. As in Fig. 13, we considered ten comoving distance bins within $5<r<100 h^{-1} \mathrm{Mpc}$, equally spaced in $\log (r)$, and integrated over halos within the redshift interval $0<z<1$. We show our results for halos above $M>5 \times$ $10^{13} h^{-1} M_{\odot}$ for DES (left error bars) and Euclid (right error bars).

these surveys, the correlation can only be measured for the full halo population and error bars become too large if one restricts to the subsample of rare massive halos above $5 \times 10^{14} h^{-1} M_{\odot}$. Nevertheless, it will be useful to measure this " $2 \ell=2$ " redshiftspace correlation in these surveys because this should tighten the constraints on cosmology. Indeed, a simultaneous analysis of $\left\langle\hat{\xi}_{R_{i}, 0}^{(s)}\right\rangle$ and $\left\langle\hat{\xi}_{R_{i}, 2}^{(s)}\right\rangle$ constrains the factor $\beta=f / \bar{b}$, and in turn the halo bias and the growth rate of density fluctuations.

\section{3. " $2 \ell=4$ " redshift-space correlation}

We show in Fig. 19 the mean " $2 \ell=4$ " redshift-space correlation $\left\langle\hat{\xi}_{R_{i}, 4}^{(s)}\right\rangle$. The signal is even weaker than for the " $2 \ell=2$ " correlation and only DES and Euclid may be able to obtain a detection. DES is unlikely to provide an accurate measure but it should still give useful upper bounds. Euclid may obtain a significant measure of the overall amplitude by gathering the information from all distance bins. This would provide an additional 
constraint on $\beta$ to the one provided by the mutipole " $2 \ell=2$ " of Fig. 18.

\section{Conclusion}

We have generalized our previous study of the real-space correlation functions of clusters of galaxies (Valageas et al. 2011) to include redshift-space distortions. On large scales they lead to an anisotropic correlation function because of the Kaiser effect, due to the correlation between density and velocity fields. There are no "fingers of God" because clusters are the largest bound objects, as opposed to galaxies that can have performed several orbital revolutions within a larger halo and show a high smallscale velocity dispersion. Then, taking into account this Kaiser effect at leading order and using a simple hierarchical model for the three-point and four-point halo correlations, we developed an analytical formalism to obtain explicit expressions for the mean redshift-space correlations and their covariance matrices. We included shot-noise and sample-variance effects as well as Gaussian and non-Gaussian contributions. This is a direct extension of the formalism presented in Valageas et al. (2011) for real-space correlations.

Expanding as usual the angular anisotropy of the redshiftspace correlation on Legendre polynomials, we considered the first three nonzero multipoles: the monopole $2 \ell=0$ (i.e. the spherically-averaged correlation), the quadrupole, $2 \ell=2$, and the next multipole, $2 \ell=4$. Higher-order multipoles should arise from nonlinear corrections to the Kaiser effect. Although they are not studied here, which would require a more complex nonlinear modeling of the Kaiser effect itself, their analysis could be performed using the formalism developped in this paper (one only needs to include higher multipoles in the sums that appear in the expressions of the covariance matrices). However, for practical purposes they are probably irrelevant for clusters of galaxies. Indeed, higher-order multipoles are increasingly difficult to measure and we found that the third mutipole, $2 \ell=4$, may only be detected (at a $1-\sigma$ level) by Euclid or a similar survey.

We obtained a reasonable agreement with numerical simulations for the mean correlations and covariance matrices on large scales $\left(r>10 h^{-1} \mathrm{Mpc}\right)$. Redshift-space distortions amplify the monopole correlation by about $10-20 \%$, depending on the halo mass. The covariance matrix is also amplified by $10-40 \%$ and the signal-to-noise ratio remains of the same order as for the real-space correlation. We found that non-Gaussian terms can contribute to the covariance matrices up to $20-60 \%$, especially for nondiagonal entries when one of the two bins corresponds to small scales $\left(\sim 10 h^{-1} \mathrm{Mpc}\right)$. They make the correlation matrices less diagonal. We also found that the correlation matrices of the quadrupole $(2 \ell=2)$ are significantly more diagonal than the correlation matrices of the monopole $(2 \ell=0)$. On the other hand, the cross-correlations of the monopole and quadrupole are quite small, on the order of 0.05 . This means that the full covariance matrix is approximately block-diagonal in the space $\left\{R_{i}, 2 \ell_{i}\right\}$, that is, we may decouple the analysis of the monopole and quadrupole.

Our predictions for ongoing and future surveys (only taking into account the sample-variance and shot-noise contributions) show that the impact of redshift distortions on the sphericalaveraged correlation (i.e. the monopole) will only be significant for DES, Erosita, and Euclid. For the other surveys investigated here, XXL, SPT, and Planck, the difference $\left\langle\hat{\xi}_{R_{i}, 0}^{(s)}\right\rangle-\left\langle\hat{\xi}_{R_{i}}\right\rangle$, between the redshift-space and real-space correlations, falls below " $1-\sigma$ " of the statistical error bar. In practice, even for these surveys it would be better to compare the data with theoretical predictions for the redshift-space correlations rather than the real-space correlations, since simple models for the latter are not more difficult to implement and one does not need a very accurate modeling of redshift distortions. For covariance matrices, it is sufficient to use the real-space ones, which are easier to compute. For DES, Erosita, and Euclid, one should take into account redshift-space distortions for both the mean correlations and their covariance matrices. However, because of their modest impact on the covariance matrices (see Figs. 3 and 4) and the limited accuracy of such computations, it is probably sufficient to use the real-space covariance matrices in a first step.

The quadrupole, $2 \ell=2$, is more difficult to measure from the distribution of clusters of galaxies, and we found that only DES, Erosita, and Euclid, among the surveys considered here, will likely provide a useful measure. In this respect, clusters are less efficient than galaxies in measuring redshift-space distortions. Even though clusters have a higher bias and stronger correlations, they are much less numerous than galaxies. This leads to a lower signal-to-noise ratio for the quadrupole. Nevertheless, such a measure would provide a nice supplement and would help tighten the constraints on cosmology. An extension of this paper would be to study on a quantitative level the expected improvement brought by this measure for these surveys. This requires a statistical analysis, using Fisher matrices or likelihood functions, which we leave for future works.

The last multipole generated at linear order by the Kaiser effect, $2 \ell=4$, is very difficult to measure from cluster surveys and we found that only Euclid will likely be able to provide a meaningful measure. Nonlinear corrections will generate higherorder multipoles but they are likely to be even more difficult to measure. Therefore, for practical purposes redshift-space distortions should only impact cluster correlations at the level of the monopole and quadrupole components.

Our results deliberately disregard observational constraints related to cluster redshifts estimation. In practice, measuring cluster redshifts is a costly task that consists in obtaining spectra for a few $(>10)$ galaxies pertaining to each cluster, leading to errors on the order of $\sigma(z)<0.01(1+z)$. This precision depends on the number of measured spectra, which in turn depends on cluster richness (and consequently, on its mass), cluster apparent size, morphological complexity, dynamical state, and distance to the observer, as well as on fixed instrumental constraints. In some cases, only the brightest central galaxy (BCG) can have its redshift measured. This may result in a biased cluster redshift estimate, for the BCG may have a small proper motion within the cluster potential well (Adami \& Ulmer 2000; Oegerle \& Hill 2001; Coziol et al. 2009). In many other cases, only photometric redshifts will be obtained, resulting in larger redshift uncertainties - on the order of $\sigma(z) \sim 0.05(1+z)$ (e.g. Pillepich et al. 2012). However, recent studies have underlined the potential of photometric surveys for studying redshift space distortions at the galaxy scale, using the information in the (2D) angular correlation function (e.g. Ross et al. 2011). We leave the application of this method to clusters to future work, and note that a quantitative analysis (e.g. a Fisher analysis) would largely benefit from a proper modeling of follow-up incompleteness, uncertainties, and biases on cluster redshifts given the halo masses and positions.

Acknowledgements. We thank M. Pierre and C. Adami for useful discussions. We thank R. Teyssier for providing us with the halo catalog from the "horizon" simulation. This work was performed using HPC resources from GENCI-CCRT (Grant 2012-t2012046803). 


\section{Appendix A: Computation of geometrical averages}

We give in this appendix the explicit expressions of the various geometrical averages of two-point correlations that are needed to compute the covariance matrix $C_{i, j}^{(s)}$, within our framework. We followed the method and the notations of Valageas et al. (2011) while we extend their results to redshift-space (especially their Appendix F).

The second term in Eq. (34) involves the average (40) of the correlation between two concentric spherical shells. Defining the $3 \mathrm{D}$ Fourier transform of the $2 \ell$-multipole top-hat as

$\tilde{W}_{3}^{(2 \ell)}(\boldsymbol{k} R)=\int_{0}^{R} \frac{\mathrm{d} \boldsymbol{r}}{4 \pi R^{3} / 3}(4 \ell+1) P_{2 \ell}\left(\mu_{\boldsymbol{r}}\right) \mathrm{e}^{\mathrm{i} \boldsymbol{k} \cdot \boldsymbol{r}}$,

where again $\mu_{\boldsymbol{r}}=\left(\boldsymbol{e}_{z} \cdot \boldsymbol{r}\right) / r$, the 3D Fourier-space window of the $i$-shell reads as

$$
\begin{aligned}
\tilde{W}_{i}^{(2 \ell)}(\boldsymbol{k}) & =\int_{\mathcal{V}_{i}} \frac{\mathrm{d} \boldsymbol{r}}{\mathcal{V}_{i}}(4 \ell+1) P_{2 \ell}\left(\mu_{\boldsymbol{r}}\right) \mathrm{e}^{\mathrm{i} \boldsymbol{k} \cdot \boldsymbol{r}} \\
& =\frac{R_{i,+}^{3} \tilde{W}_{3}^{(2 \ell)}\left(\boldsymbol{k} R_{i,+}\right)-R_{i,-}^{3} \tilde{W}_{3}^{(2 \ell)}\left(\boldsymbol{k} R_{i,-}\right)}{R_{i,+}^{3}-R_{i,-}^{3}} .
\end{aligned}
$$

In Eq. (A.1) the subscript 3 recalls that we consider a 3D window. To simplify notations we did not recall this fact in the window $\tilde{W}_{i}^{(2 \ell)}$ associated with each 3D radial bin. In studies of the real-space correlation (Valageas et al. 2011), only the usual tophat window $\tilde{W}_{3}^{(0)}(k R)$ appears, which does not depend on the angle of $\boldsymbol{k}$. Here, because we study the anisotropic redshift-space correlation and its various multipoles (25) higher-order multipoles $\tilde{W}_{3}^{(2 \ell)}(\boldsymbol{k} R)$ appear, which depend on the direction of $\boldsymbol{k}$. Equation (A.1) also reads as

$\tilde{W}_{3}^{(2 \ell)}(\boldsymbol{k} R)=\hat{W}_{3}^{(2 \ell)}(k R) P_{2 \ell}\left(\mu_{k}\right)$,

where the angular dependence has been factored out, with

$\hat{W}_{3}^{(2 \ell)}(k R)=(-1)^{\ell}(4 \ell+1) \int_{0}^{R} \frac{\mathrm{d} r 3 r^{2}}{R^{3}} j_{2 \ell}(k r)$.

This yields for the window of the $i$-shell,

$\tilde{W}_{i}^{(2 \ell)}(\boldsymbol{k})=\hat{W}_{i}^{(2 \ell)}(k) P_{2 \ell}\left(\mu_{k}\right)$,

with

$\hat{W}_{i}^{(2 \ell)}(k)=\frac{R_{i,+}^{3} \hat{W}_{3}^{(2 \ell)}\left(k R_{i,+}\right)-R_{i,-}^{3} \hat{W}_{3}^{(2 \ell)}\left(k R_{i,-}\right)}{R_{i,+}^{3}-R_{i,-}^{3}}$.

In practice, the redshift-space correlation of clusters of galaxies should be dominated by the Kaiser effect and the lower multipoles $2 \ell=0,2$, and 4 as in Eqs. (11)-(14). Therefore, we only give explicit expressions for low-order multipoles, which read as

$$
\begin{aligned}
\hat{W}_{3}^{(0)}(k R)= & 3 \frac{\sin (k R)-k R \cos (k R)}{(k R)^{3}}, \\
\hat{W}_{3}^{(2)}(k R)= & \frac{-15}{(k R)^{3}}[k R \cos (k R)-4 \sin (k R)+3 \operatorname{Si}(k R)], \\
\hat{W}_{3}^{(4)}(k R)= & \frac{27}{2(k R)^{5}}\left[\left(105-2(k R)^{2}\right) k R \cos (k R)\right. \\
& \left.-\left(105-22(k R)^{2}\right) \sin (k R)+15(k R)^{2} \operatorname{Si}(k R)\right],
\end{aligned}
$$

where $\operatorname{Si}(z)=\int_{0}^{z} \mathrm{~d} t \sin (t) / t$ is the sine integral. Then, writing the two-point correlation function in Eq. (40) in terms of the power spectrum (9), the angular integration gives

$$
\begin{aligned}
\overline{\xi_{i^{\prime}, j^{\prime}}^{(s)}}= & \int_{0}^{\infty} \frac{\mathrm{d} k}{k} \Delta^{2}(k, z) \hat{W}_{i}^{\left(2 \ell_{i}\right)}(k) \hat{W}_{j}^{\left(2 \ell_{j}\right)}(k) \\
& \times\left\{\left(1+\frac{2 \beta}{3}+\frac{\beta^{2}}{5}\right)\left(\begin{array}{ccc}
0 & 2 \ell_{i} & 2 \ell_{j} \\
0 & 0 & 0
\end{array}\right)^{2}+\left(\frac{4 \beta}{3}+\frac{4 \beta^{2}}{7}\right)\right. \\
& \left.\times\left(\begin{array}{ccc}
2 & 2 \ell_{i} & 2 \ell_{j} \\
0 & 0 & 0
\end{array}\right)^{2}+\frac{8 \beta^{2}}{35}\left(\begin{array}{ccc}
4 & 2 \ell_{i} & 2 \ell_{j} \\
0 & 0 & 0
\end{array}\right)^{2}\right\}
\end{aligned}
$$

Next, the last term in Eq. (34) involves the non-connected fourpoint average

$$
\begin{aligned}
\overline{\xi_{i ; j^{\prime}}^{(s)} \xi_{i^{\prime} ; j}^{(s)}}= & \int \frac{\mathrm{d} \chi_{j}}{\mathcal{D}_{i}} \int \frac{\mathrm{d} \boldsymbol{\Omega}_{i} \mathrm{~d} \boldsymbol{\Omega}_{j}}{(\Delta \Omega)^{2}} \int_{i} \frac{\mathrm{d} s_{i^{\prime}}}{\mathcal{V}_{i}} \int_{j} \frac{\mathrm{d} \boldsymbol{s}_{j^{\prime}}}{\mathcal{V}_{j}}\left(4 \ell_{i}+1\right) \\
& \times\left(4 \ell_{j}+1\right) P_{2 \ell_{i}}\left(\mu_{i^{\prime}}\right) P_{2 \ell_{j}}\left(\mu_{j^{\prime}}\right) \xi_{i ; j^{\prime}}^{(s)} \xi_{i^{\prime} ; j}^{(s)} .
\end{aligned}
$$

As in Valageas et al. (2011), expressing the two-point correlation functions in terms of the power spectrum, using the flat-sky (small angle) approximation, as well as Limber's approximation (Limber 1953; Kaiser 1992; Munshi et al. 2008), we obtain

$$
\begin{aligned}
\overline{\xi_{i ; j^{\prime}}^{(s)} \xi_{i^{\prime} ; j}^{(s)}}= & \frac{2 \pi}{\mathcal{D}} \int \mathrm{d} \boldsymbol{k}_{1} \mathrm{~d} \boldsymbol{k}_{2} P\left(k_{1}\right)\left(1+\beta \mu_{1}^{2}\right)^{2} P\left(k_{2}\right)\left(1+\beta \mu_{2}^{2}\right)^{2} \\
& \times \delta_{D}\left(k_{1 \|}+k_{2 \|}\right) \tilde{W}_{i}^{\left(2 \ell_{i}\right)}\left(\boldsymbol{k}_{1}\right) \tilde{W}_{j}^{\left(2 \ell_{j}\right)}\left(\boldsymbol{k}_{2}\right) \\
& \times \tilde{W}_{2}\left(\left|\boldsymbol{k}_{1 \perp}+\boldsymbol{k}_{2 \perp}\right| \mathcal{D} \theta_{\mathrm{s}}\right)^{2},
\end{aligned}
$$

where $\mu_{i}=\left(\boldsymbol{e}_{z} \cdot \boldsymbol{k}_{i}\right) / k_{i}, \theta_{\mathrm{s}}$ is the angular radius of the survey window (in the flat-sky approximation),

$(\Delta \Omega)=\pi \theta_{\mathrm{s}}^{2}$,

and $\tilde{W}_{2}$ is the $2 \mathrm{D}$ Fourier-space circular window,

$\tilde{W}_{2}\left(k_{\perp} \mathcal{D} \theta_{\mathrm{S}}\right)=\int \frac{\mathrm{d} \boldsymbol{\theta}}{\pi \theta_{\mathrm{S}}^{2}} \mathrm{e}^{\mathrm{i} k_{\perp} \cdot \mathcal{D} \theta}=\frac{2 J_{1}\left(k_{\perp} \mathcal{D} \theta_{\mathrm{S}}\right)}{k_{\perp} \mathcal{D} \theta_{\mathrm{S}}}$.

Introducing an auxiliary wavenumber $\boldsymbol{k}_{\perp}$ with a Dirac factor $\int \mathrm{d} \boldsymbol{k}_{\perp} \delta_{D}\left(\boldsymbol{k}_{\perp}-\boldsymbol{k}_{1 \perp}-\boldsymbol{k}_{2 \perp}\right)$ and using the exponential representation of Dirac functions, we can partially factorize the integrals and perform a few angular integrations. This yields

$\overline{\xi_{i ; j^{\prime}}^{(s)} \xi_{i^{\prime} ; j}^{(s)}}=\int \frac{\mathrm{d} \boldsymbol{r}}{2 \pi r \mathcal{D}^{2} \theta_{\mathrm{s}}} \mathcal{I}_{i, 2 \ell_{i}}^{(s)}(\boldsymbol{r}) \mathcal{I}_{j, 2 \ell_{j}}^{(s)}(\boldsymbol{r}) A^{(s)}\left(\frac{\boldsymbol{r}}{\mathcal{D} \theta_{\mathrm{s}}}\right)$,

where we introduced

$A^{(s)}\left(\frac{\boldsymbol{r}}{\mathcal{D} \theta_{\mathrm{s}}}\right)=\frac{r \mathcal{D} \theta_{\mathrm{s}}}{2 \pi} \int \mathrm{d} \boldsymbol{k}_{\perp} \mathrm{e}^{\mathrm{i} \boldsymbol{r}_{\perp} \cdot \boldsymbol{k}_{\perp}} \tilde{W}_{2}\left(k_{\perp} \mathcal{D} \theta_{\mathrm{s}}\right)^{2}$

and

$\mathcal{I}_{i .2 \ell_{i}}^{(s)}(\boldsymbol{r})=\int \mathrm{d} \boldsymbol{k} \mathrm{e}^{\mathrm{i} \boldsymbol{k} \cdot \boldsymbol{r}} P(k)\left(1+\beta \mu_{\boldsymbol{k}}^{2}\right)^{2} \tilde{W}_{i}^{\left(2 \ell_{i}\right)}(\boldsymbol{k})$.

Using the multipole decomposition (11) and the expansion of plane waves on spherical harmonics, we can write $\mathcal{I}_{i, 2 \ell_{i}}^{(s)}(\boldsymbol{r})$ as

$\mathcal{I}_{i, 2 \ell_{i}}^{(s)}(\boldsymbol{r})=\sum_{\ell=0}^{4} \mathcal{I}_{i, 2 \ell_{i}}^{(s ; 2 \ell)}(r) P_{2 \ell}\left(\mu_{\boldsymbol{r}}\right)$,

where the sum over $\ell$ runs up to 4 because the expansion (11) only includes the terms $0 \leq \ell \leq 2$ and we only study estimators $\hat{\xi}_{R_{i}, 2 \ell_{i}}^{(s)}$ with $0 \leq \ell_{i} \leq 2$. If we consider a model where the 
redshift-space correlation includes multipoles up to $2 \ell_{\max }$ and we study estimators up to order $2 \hat{\ell}_{\max }$ the sum (A.18) runs up to $\ell \leq \ell_{\max }+\hat{\ell}_{\max }$. Here we introduced

$$
\begin{aligned}
\mathcal{I}_{i, 2 \ell_{i}}^{(s ; 2 \ell)}(r)= & (4 \ell+1) \sum_{\ell^{\prime}=0}^{2} \int_{0}^{\infty} \frac{\mathrm{d} x x \xi^{\left(s ; 2 \ell^{\prime}\right)}(x)}{r\left(R_{i,+}^{3}-R_{i,-}^{3}\right)} \\
& \times\left[R_{i,+}^{2} \mathcal{W}_{\ell_{i}}^{\left(\ell, \ell^{\prime}\right)}\left(\frac{r}{R_{i,+}}, \frac{x}{R_{i,+}}\right)\right. \\
& \left.-R_{i,-}^{2} W_{\ell_{i}}^{\left(\ell, \ell^{\prime}\right)}\left(\frac{r}{R_{i,-}}, \frac{x}{R_{i,-}}\right)\right],
\end{aligned}
$$

and

$$
\begin{aligned}
& \mathcal{W}_{\ell_{i}}^{\left(\ell, \ell^{\prime}\right)}(a, b)=(-1)^{\ell+\ell^{\prime}}\left(\begin{array}{ccc}
2 \ell & 2 \ell^{\prime} & 2 \ell_{i} \\
0 & 0 & 0
\end{array}\right)^{2} \frac{2 a b}{\pi} \\
& \times \int_{0}^{\infty} \mathrm{d} u u^{2} \hat{W}_{3}^{\left(2 \ell_{i}\right)}(u) j_{2 \ell}(a u) j_{2 \ell^{\prime}}(b u) .
\end{aligned}
$$

Then, substituting the expansion (A.18) into Eq. (A.15) we obtain

$$
\begin{aligned}
\overline{\xi_{i ; j^{\prime}}^{(s)} \xi_{i^{\prime} ; j}^{(s)}}= & 2 \theta_{\mathrm{s}} \int_{0}^{\infty} \frac{\mathrm{d} r r}{\left(\mathcal{D} \theta_{\mathrm{s}}\right)^{2}} \sum_{\ell, \ell^{\prime}=0}^{4} \mathcal{I}_{i, 2 \ell_{i}}^{(s ; 2 \ell)}(r) \mathcal{I}_{j, 2 \ell_{j}}^{\left(s ; 2 \ell^{\prime}\right)}(r) \\
& \times A^{\left(\ell, \ell^{\prime}\right)}\left(\frac{r}{\mathcal{D} \theta_{\mathrm{s}}}\right),
\end{aligned}
$$

with

$A^{\left(\ell, \ell^{\prime}\right)}(y)=\frac{1}{2} \int_{-1}^{1} \mathrm{~d} \mu P_{2 \ell}(\mu) P_{2 \ell^{\prime}}(\mu) A^{(s)}\left(y, \mu^{2}\right)$,

where we used that $A^{(s)}$ defined in Eq. (A.16) also writes as

$A^{(s)}(\boldsymbol{y}) \equiv A^{(s)}\left(y, \mu^{2}\right)=y \int_{0}^{\infty} \mathrm{d} u u \tilde{W}_{2}(u)^{2} J_{0}\left(y u \sqrt{1-\mu^{2}}\right)$.

These expressions closely follow those obtained in Valageas et al. (2011). To recover their real-space results, it is sufficient to set $\beta=0$ in Eq. (9), which makes the power spectrum isotropic so that only monopole contributions (i.e., $\ell=0$ ) remain. Thus, in Eq. (A.21) there only remains the term $\ell=\ell^{\prime}=0$, and the function $A^{(0,0)}(y)$ can actually be computed explicitly (see their Eqs. (F10) and (F11)).

As in Valageas et al. (2011), we preferred to use the configuration-space expression (A.21) rather than the Fourierspace expression (A.12) for numerical computations. Indeed, the integrals have been partially factored out in Eq. (A.21) (so that it has the same level of complexity as a two-dimensional integral) and the oscillatory kernels $\tilde{W}_{i}^{\left(2 \ell_{i}\right)}$ of Eq. (A.12) have been replaced by the slowly-varying kernels $\mathcal{W}_{\ell_{i}}^{\left(\ell, \ell^{\prime}\right)}$ of Eq. (A.19), see Eqs. (B.17)-(B.20).

We now turn to the contribution (38) that arises from the three-point correlation function. The first term within the brackets in Eq. (38) involves the product of two averages $\overline{\xi_{i^{\prime}}^{(s)}}$ and $\overline{\xi_{j^{\prime}}^{(s)}}$, which are given in Eq. (27). Following the analysis of Valageas et al. (2011), the second term can be written in configuration space as

$\overline{\xi_{i^{\prime}, i}^{(s)} \xi_{i^{\prime}, j^{\prime}}^{(s)}}=\int_{\mathcal{V}_{i}} \frac{\mathrm{d} \boldsymbol{r}}{\mathcal{V}_{i}}\left(4 \ell_{i}+1\right) P_{2 \ell_{i}}\left(\mu_{\boldsymbol{r}}\right) \xi^{(s)}(\boldsymbol{r}) \mathcal{I}_{j, 2 \ell_{j}}^{(s)}(\boldsymbol{r})$.
Using the expansions (11) and (A.18), this yields

$$
\begin{aligned}
\overline{\xi_{i^{\prime}, i}^{(s)} \xi_{i^{\prime}, j^{\prime}}^{(s)}}= & \left(4 \ell_{i}+1\right) \int_{R_{i,-}}^{R_{i,+}} \frac{\mathrm{d} r 3 r^{2}}{R_{i,+}^{3}-R_{i,-}^{3}} \sum_{\ell=0}^{2} \sum_{\ell^{\prime}=0}^{4} \xi^{(s ; 2 \ell)}(r) \\
& \times \mathcal{I}_{j, 2 \ell_{j}}^{\left(s ; 2 \ell^{\prime}\right)}(r)\left(\begin{array}{ccc}
2 \ell & 2 \ell^{\prime} & 2 \ell_{i} \\
0 & 0 & 0
\end{array}\right)^{2} .
\end{aligned}
$$

The third term within the brackets in Eq. (38) is obtained from Eq. (A.25) by exchanging the labels " $i$ " and " $j$ ".

We now turn to the contribution (39) that arises from the four-point correlation function, proceeding in a similar fashion. The new geometrical average involved by the first two terms within the brackets in Eq. (39) reads as

$$
\begin{aligned}
\overline{\xi_{i, j} \xi_{i ; j^{\prime}}^{(s)}}= & 2 \theta_{\mathrm{s}} \int_{0}^{\infty} \frac{\mathrm{d} r r}{\left(\mathcal{D} \theta_{\mathrm{s}}\right)^{2}} \sum_{\ell=0}^{2} \sum_{\ell^{\prime}=0}^{4} \xi^{(s ; 2 \ell)}(r) \mathcal{I}_{j, 2 \ell_{j}}^{\left(s ; 2 \ell^{\prime}\right)}(r) \\
& \times A^{\left(\ell, \ell^{\prime}\right)}\left(\frac{r}{\mathcal{D} \theta_{\mathrm{s}}}\right),
\end{aligned}
$$

which does not depend on the bin $i$. The third term involves the cylindrical average

$\overline{\xi_{\text {cyl }}}=\pi \int_{0}^{\infty} \frac{\mathrm{d} k}{k} \frac{\Delta^{2}(k)}{\mathcal{D} k} \tilde{W}_{2}\left(k \mathcal{D} \theta_{\mathrm{s}}\right)^{2}$.

The fourth term, which does not factor, gives rise to

$$
\begin{aligned}
\overline{\xi_{j^{\prime} ; i}^{(s)} \xi_{i ; j} \xi_{j ; i^{\prime}}^{(s)}}= & 2 \theta_{\mathrm{s}} \int_{0}^{\infty} \frac{\mathrm{d} r r}{\left(\mathcal{D} \theta_{\mathrm{s}}\right)^{2}} \sum_{\ell=0}^{2} \sum_{\ell^{\prime}, \ell^{\prime \prime}=0}^{4} \xi^{(s ; 2 \ell)}(r) \\
& \times \mathcal{I}_{i, 2 \ell_{i}}^{\left(s ; 2 \ell^{\prime}\right)}(r) \mathcal{I}_{j, 2 \ell_{j}}^{\left(s ; 2 \ell^{\prime \prime}\right)}(r) A^{\left(\ell, \ell^{\prime}, \ell^{\prime \prime}\right)}\left(\frac{r}{\mathcal{D} \theta_{\mathrm{s}}}\right),
\end{aligned}
$$

where we introduced, as in Eq. (A.22),

$A^{\left(\ell, \ell^{\prime}, \ell^{\prime \prime}\right)}(y)=\frac{1}{2} \int_{-1}^{1} \mathrm{~d} \mu P_{2 \ell}(\mu) P_{2 \ell^{\prime}}(\mu) P_{2 \ell^{\prime \prime}}(\mu) A^{(s)}\left(y, \mu^{2}\right)$.

The last two terms within the brackets in Eq. (39) involve the geometrical average

$$
\begin{aligned}
\overline{\xi_{j^{\prime} ; i}^{(s)} \xi_{i, i^{\prime}}^{(s)} \xi_{i^{\prime} ; j}^{(s)}}= & \frac{\theta_{\mathrm{s}}}{2 \pi} \int \frac{\mathrm{d} \boldsymbol{r}}{r\left(\mathcal{D} \theta_{\mathrm{s}}\right)^{2}} \int_{\mathcal{V}_{i}} \frac{\mathrm{d} \boldsymbol{r}^{\prime}}{\mathcal{V}_{i}}\left(4 \ell_{i}+1\right) P_{2 \ell_{i}}\left(\mu_{\boldsymbol{r}^{\prime}}\right) \\
& \times \mathcal{I}_{j, 2 \ell_{j}}^{(s)}(\boldsymbol{r}) \xi^{(s)}\left(\boldsymbol{r}^{\prime}\right) \xi^{(s)}\left(\boldsymbol{r}+\boldsymbol{r}^{\prime}\right) A^{(s)}\left(\frac{\boldsymbol{r}}{\mathcal{D} \theta_{\mathrm{s}}}\right) .
\end{aligned}
$$

To integrate over angles, we again introduce a Dirac factor $\int \mathrm{d} \boldsymbol{r}^{\prime \prime} \delta_{D}\left(\boldsymbol{r}^{\prime \prime}-\boldsymbol{r}-\boldsymbol{r}^{\prime}\right)$ that we write under its exponential representation, which we expand over spherical harmonics. Using the expansions (11) and (A.18), we obtain

$$
\begin{aligned}
\overline{\xi_{j^{\prime} ;}^{(s)} \xi_{i, i^{\prime}}^{(s)} \xi_{i^{\prime} ; j}^{(s)}}= & \theta_{\mathrm{s}} \int_{0}^{\infty} \frac{\mathrm{d} r r}{\left(\mathcal{D} \theta_{\mathrm{s}}\right)^{2}} \int_{R_{i,-}}^{R_{i,+}} \frac{\mathrm{d} r^{\prime} 3 r^{\prime 2}}{R_{i,+}^{3}-R_{i,-}^{3}} \int_{\left|r-r^{\prime}\right|}^{r+r^{\prime}} \frac{\mathrm{d} r^{\prime \prime} r^{\prime \prime}}{r r^{\prime}} \\
& \times \sum_{\ell=0}^{4} \sum_{\ell^{\prime}, \ell^{\prime \prime}=0}^{2} \mathcal{I}_{j, 2 \ell}^{(s ; 2 \ell)}(r) \xi^{\left(s ; 2 \ell^{\prime}\right)}\left(r^{\prime}\right) \xi^{\left(s ; 2 \ell^{\prime \prime}\right)}\left(r^{\prime \prime}\right) \sum_{n^{\prime}=\left|\ell_{i}-\ell^{\prime}\right|}^{\ell_{i}+\ell^{\prime}} \\
& \times \sum_{n=\left|n^{\prime}-\ell^{\prime \prime}\right|}^{n^{\prime}+\ell^{\prime \prime}}(-1)^{n+n^{\prime}+\ell^{\prime \prime}}\left(4 \ell_{i}+1\right)(4 n+1)\left(4 n^{\prime}+1\right) \\
& \times\left(\begin{array}{ccc}
2 n^{\prime} & 2 \ell_{i} & 2 \ell^{\prime} \\
0 & 0 & 0
\end{array}\right)^{2}\left(\begin{array}{ccc}
2 n & 2 n^{\prime} & 2 \ell^{\prime \prime} \\
0 & 0 & 0
\end{array}\right)^{2} A^{(\ell, n)}\left(\frac{r}{\mathcal{D} \theta_{\mathrm{s}}}\right) \\
& \times C^{n, n^{\prime}, \ell^{\prime \prime}}\left(r, r^{\prime}, r^{\prime \prime}\right),
\end{aligned}
$$


where we introduced the symmetric functions

$$
\begin{aligned}
C^{\ell_{1}, \ell_{2}, \ell_{3}}\left(r_{1}, r_{2}, r_{3}\right)= & \frac{4}{\pi} r_{1} r_{2} r_{3} \int_{0}^{\infty} \mathrm{d} k k^{2} j_{2 \ell_{1}}\left(k r_{1}\right) \\
& \times j_{2 \ell_{2}}\left(k r_{2}\right) j_{2 \ell_{3}}\left(k r_{3}\right) .
\end{aligned}
$$

For numerical computations it is more efficient to write Eq. (A.31) in a partially factorized form by exchanging the order of the integrations and moving the integration over $k$ included in the function $C^{\ell_{1}, \ell_{2}, \ell_{3}}$ to the left. Using the decomposition (11)-(14) and integrating over $r^{\prime \prime}$, this gives

$$
\begin{aligned}
& \overline{\xi_{j^{\prime} ; i}^{(s)} \xi_{i, i^{\prime}}^{(s)} \xi_{i^{\prime} ; j}^{(s)}}=2 \theta_{\mathrm{s}} \int_{0}^{\infty} \frac{\mathrm{d} k}{k} \Delta^{2}(k) \sum_{\ell=0}^{4} \sum_{\ell^{\prime}, \ell^{\prime \prime}=0}^{2} \sum_{n^{\prime}=\left|\ell_{i}-\ell^{\prime}\right|}^{\ell_{i}+\ell^{\prime}} \sum_{n=\left|n^{\prime}-\ell^{\prime \prime}\right|}^{n^{\prime}+\ell^{\prime \prime}} \\
& \times(-1)^{n+n^{\prime}+\ell^{\prime \prime}}\left(4 \ell_{i}+1\right)(4 n+1)\left(4 n^{\prime}+1\right)\left(\begin{array}{ccc}
2 n^{\prime} & 2 \ell_{i} & 2 \ell^{\prime} \\
0 & 0 & 0
\end{array}\right)^{2} \\
& \times\left(\begin{array}{ccc}
2 n & 2 n^{\prime} & 2 \ell^{\prime \prime} \\
0 & 0 & 0
\end{array}\right)^{2} F^{\left(2 \ell^{\prime \prime}\right)} \int_{0}^{\infty} \frac{\mathrm{d} r r}{\left(\mathcal{D} \theta_{\mathrm{s}}\right)^{2}} j_{2 n}(k r) \mathcal{I}_{j ; 2 \ell_{j}}^{(s ; 2 \ell)}(r) \\
& \times A^{(\ell, n)}\left(\frac{r}{\mathcal{D} \theta_{\mathrm{s}}}\right) \int_{R_{i,-}}^{R_{i,+}} \frac{\mathrm{d} r^{\prime} 3 r^{\prime 2}}{R_{i,+}^{3}-R_{i,-}^{3}} j_{2 n^{\prime}}\left(k r^{\prime}\right) \xi^{\left(s ; 2 \ell^{\prime}\right)}\left(r^{\prime}\right),
\end{aligned}
$$

where $F^{\left(2 \ell^{\prime \prime}\right)}$ is the prefactor in Eqs. (12)-(14),

$F^{(0)}=1+\frac{2 \beta}{3}+\frac{\beta^{2}}{5}, F^{(2)}=-\frac{4 \beta}{3}-\frac{4 \beta^{2}}{7}, F^{(4)}=\frac{8 \beta^{2}}{35}$.

Again, all these geometrical averages of redshift-space correlations reduce to the real-space results of Valageas et al. (2011), if we set $\beta=0$ and only keep the monopole terms.

\section{Appendix B: Some useful integrals}

We describe here the computation of some of the functions introduced in Appendix A.

\section{B.1. Multipoles of $A^{(s)}(y)$}

Using the expression (A.23) and the expansion (Gradshteyn \& Ryzhik 1994)

$$
\begin{aligned}
J_{0}\left(z \sqrt{1-\mu^{2}}\right)= & \sqrt{\frac{2 \pi}{z}} \sum_{n=0}^{\infty}(2 n+1 / 2) \frac{(2 n-1) ! !}{2^{n} n !} \\
& \times J_{2 n+1 / 2}(z) P_{2 n}(\mu),
\end{aligned}
$$

we can write

$A^{(s)}\left(y, \mu^{2}\right)=\sum_{n=0}^{\infty}(4 n+1) \frac{(2 n-1) ! !}{2^{n} n !} B_{n}(y) P_{2 n}(\mu)$,

with

$B_{n}(y)=y \int_{0}^{\infty} \mathrm{d} u u \tilde{W}_{2}(u)^{2} j_{2 n}(y u)$.

This yields the multipoles

$$
\begin{aligned}
A^{\left(\ell_{1}, \ell_{2}\right)}(y)= & \sum_{n=\left|\ell_{1}-\ell_{2}\right|}^{\ell_{1}+\ell_{2}}(4 n+1) \frac{(2 n-1) ! !}{2^{n} n !}\left(\begin{array}{ccc}
2 \ell_{1} & 2 \ell_{2} & 2 n \\
0 & 0 & 0
\end{array}\right)^{2} \\
& \times B_{n}(y),
\end{aligned}
$$

and

$$
A^{\left(\ell_{1}, \ell_{2}, \ell_{3}\right)}(y)=\sum_{n=0}^{\ell_{1}+\ell_{2}+\ell_{3}}(4 n+1) \frac{(2 n-1) ! !}{2^{n} n !} Y^{\ell_{1}, \ell_{2}, \ell_{3}, n} B_{n}(y),
$$

with

$$
Y^{\ell_{1}, \ell_{2}, \ell_{3}, \ell_{4}}=\frac{1}{2} \int_{-1}^{1} \mathrm{~d} \mu P_{2 \ell_{1}}(\mu) P_{2 \ell_{2}}(\mu) P_{2 \ell_{3}}(\mu) P_{2 \ell_{4}}(\mu) .
$$

Because we only considered the low-order multipoles $0 \leq \ell_{i} \leq$ 2 of the redshift-space correlation function, we only need the first few multipoles $A^{\left(\ell_{1}, \ell_{2}\right)}$ and $A^{\left(\ell_{1}, \ell_{2}, \ell_{3}\right)}$. The associated numbers $Y^{\ell_{1}, \ell_{2}, \ell_{3}, \ell_{4}}$ and functions $B_{n}(y)$ can be computed in advance.

\section{B.2. Symmetric functions $C^{\ell_{1}, \ell_{2}, \ell_{3}}$}

The functions $C^{\ell_{1}, \ell_{2}, \ell_{3}}$ defined in Eq. (A.32) vanish if the three lengths $\left\{r_{1}, r_{2}, r_{3}\right\}$ do not obey triangular inequalities. It is also useful to note that these functions obey the scale invariance

$\lambda>0: \quad C^{\ell_{1}, \ell_{2}, \ell_{3}}\left(\lambda r_{1}, \lambda r_{2}, \lambda r_{3}\right)=C^{\ell_{1}, \ell_{2}, \ell_{3}}\left(r_{1}, r_{2}, r_{3}\right)$.

For $\left|r_{1}-r_{2}\right|<r_{3}<r_{1}+r_{2}$, as in Eq. (A.31), we obtain explicit expressions using the recursion relations of Bessel functions and the property (Gradshteyn \& Ryzhik 1994)

$$
\begin{aligned}
\int_{0}^{\infty} \mathrm{d} k k^{1-\alpha} J_{\alpha}(a k) J_{\beta}(b k) J_{\beta}(c k)= & \frac{(b c)^{\alpha-1}}{\sqrt{2 \pi} a^{\alpha}}(\sin v)^{\alpha-1 / 2} \\
& \times P_{\beta-1 / 2}^{1 / 2-\alpha}(\cos v),
\end{aligned}
$$

for

$|a-b|<c<a+b, \quad 2 b c \cos (v)=b^{2}+c^{2}-a^{2}$.

Indeed, Eq. (A.32) also reads as

$$
\begin{aligned}
C^{\ell_{1}, \ell_{2}, \ell_{3}}\left(r_{1}, r_{2}, r_{3}\right)= & \sqrt{2 \pi r_{1} r_{2} r_{3}} \int_{0}^{\infty} \mathrm{d} k \sqrt{k} J_{2 \ell_{1}+1 / 2}\left(k r_{1}\right) \\
& \times J_{2 \ell_{2}+1 / 2}\left(k r_{2}\right) J_{2 \ell_{3}+1 / 2}\left(k r_{3}\right) .
\end{aligned}
$$

Then, using the recursion

$J_{2 \ell+1 / 2}(z)=\frac{4 \ell-1}{z} J_{2 \ell-1 / 2}(z)-J_{2 \ell-3 / 2}(z)$,

we can lower the indices $\left\{\ell_{1}, \ell_{2}, \ell_{3}\right\}$ and can express all required quantities in terms of integrals of the form (B.8) with two identical indices and the right power-law prefactor. For instance, the first few functions read as

$C^{0,0,0}=1$,

$C^{0,1,1}=\frac{3 \mu_{1}^{2}-1}{2}$

$C^{1,1,1}=\frac{3}{2} \frac{r_{2} r_{3}}{r_{1}^{2}} \mu_{1}\left(1-\mu_{1}^{2}\right)-\frac{3 \mu_{1}^{2}-1}{2}$,

were we defined

$\mu_{1}=\frac{r_{2}^{2}+r_{3}^{2}-r_{1}^{2}}{2 r_{2} r_{3}}$. 

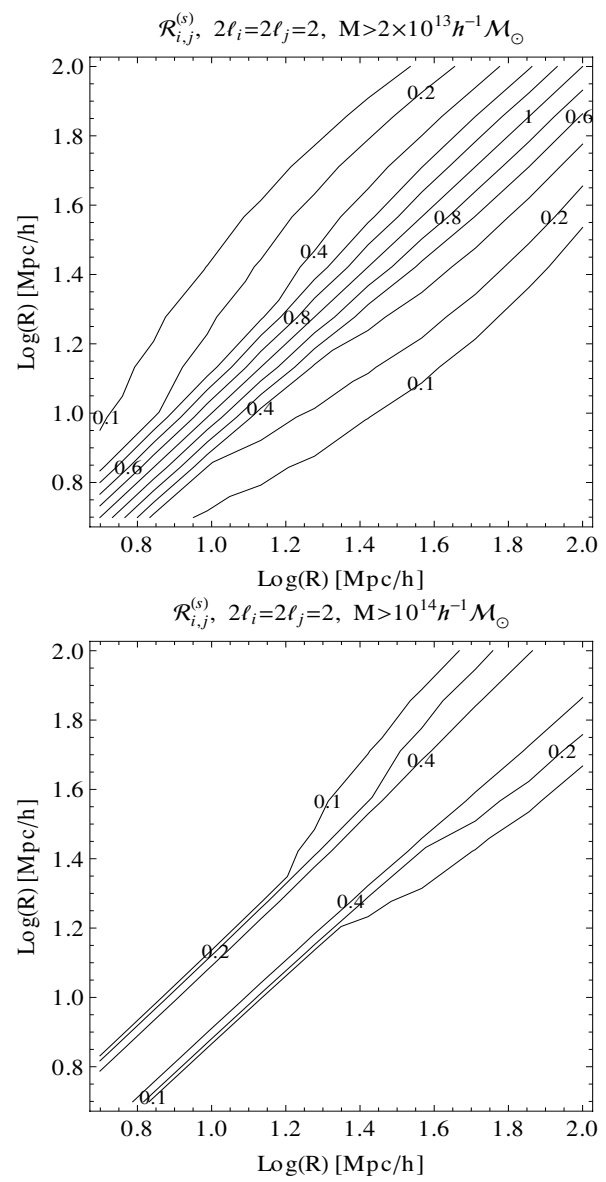

Fig. C.1. Contour plots for the redshift-space auto-correlation matrices $\mathcal{R}_{i, j}^{(s)}$, defined as in Eq. (41), for the " $2 \ell=2$ " redshift-space correlation studied in Sect. 4.3. We considered the same cases (distance bins, redshift interval, and mass thresholds) as in Fig. 8, where we plotted the auto-correlation matrices of the monopole term of the redshift-space correlation.

\section{B.3. Functions $\mathcal{W}_{\ell_{i}}^{\ell, \ell^{\prime}}(a, b)$}

To compute the geometrical means $\mathcal{I}_{i, 2 \ell_{i}}^{(s)}(\boldsymbol{r})$ of the redshift-space correlation with respect to bin $i$, defined in Eq. (A.17), we need the functions $\mathcal{W}_{\ell_{i}}^{\ell, \ell^{\prime}}$ defined in Eq. (A.20). Using Eq. (A.4), they also write as

$$
\begin{aligned}
\mathcal{W}_{\ell_{i}}^{\left(\ell, \ell^{\prime}\right)}(a, b)= & (-1)^{\ell+\ell^{\prime}+\ell_{i}}\left(4 \ell_{i}+1\right)\left(\begin{array}{ccc}
2 \ell & 2 \ell^{\prime} & 2 \ell_{i} \\
0 & 0 & 0
\end{array}\right)^{2} \\
& \times \int_{0}^{1} \mathrm{~d} r \frac{3 r}{2} C^{\ell, \ell^{\prime}, \ell_{i}}(a, b, r),
\end{aligned}
$$

where the functions $C^{\ell_{1}, \ell_{2}, \ell_{3}}$ were defined in Eq. (A.32). From the triangular constraint on these functions $C^{\ell_{1}, \ell_{2}, \ell_{3}}$ we obtain

$|a-b|>1: \quad \mathcal{W}_{\ell_{i}}^{\left(\ell, \ell^{\prime}\right)}=0$,

while the scale invariance (B.7) leads to

$a+b \leq 1: \mathcal{W}_{\ell_{i}}^{\left(\ell, \ell^{\prime}\right)}(a, b)=(a+b)^{2} \mathcal{W}_{\ell_{i}}^{\left(\ell, \ell^{\prime}\right)}\left(\frac{a}{a+b}, \frac{b}{a+b}\right)$.

Then, from the explicit expressions obtained in Appendix B.2, which are rational functions of $\left\{r_{1}, r_{2}, r_{3}\right\}$, we can perform the
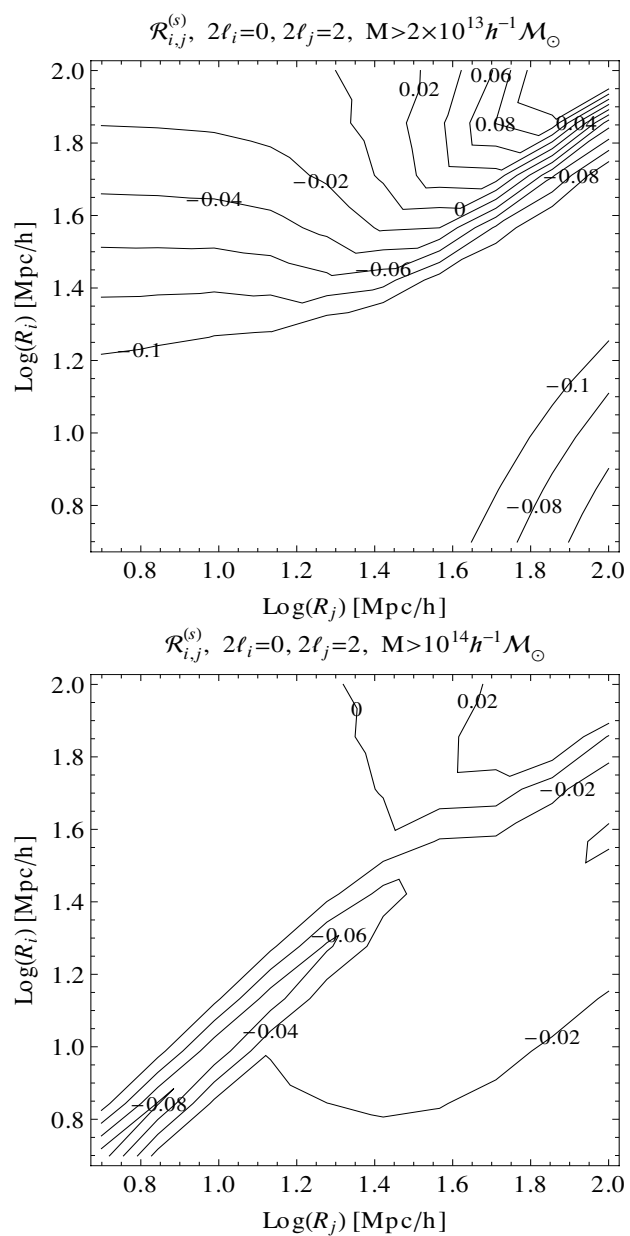

Fig. C.2. Contour plots for the redshift-space cross-correlation matrices $\mathcal{R}_{i, j}^{(s)}$, defined as in Eq. (C.1), for the " $2 \ell_{i}=0$ " and " $2 \ell_{j}=2$ " redshiftspace correlations. We considered the same cases (distance bins, redshift interval, and mass thresholds) as in Figs. 8 and C.1.

integral (B.16) and derive explicit expressions. In particular, the first function reads as

$$
\begin{array}{ll}
|a-b|<1, a+b<1: & \mathcal{W}_{0}^{(0,0)}=3 a b \\
|a-b|<1, a+b>1: & \mathcal{W}_{0}^{(0,0)}=\frac{3}{4}\left[1-(a-b)^{2}\right] .
\end{array}
$$

\section{Appendix C: Correlation matrices of the " $2 \ell=2$ " redshift-space correlation}

\section{C.1. Auto-correlation of the " $2 \ell=2$ " multipole}

We show in Fig. C. 1 the auto-correlation matrices of the " $2 \ell=$ 2 " redshift-space correlation. For simplicity we only plot our results for the full matrices that include the non-Gaussian contributions associated with the 3-pt and 4-pt correlation functions. We can see that they are significantly more diagonal than the autocorrelation matrices of the monopole term that were displayed in the right panels of Fig. 8 . 


\section{C.2. Cross-correlation of the " $2 \ell=2$ " and " $2 \ell=2$ " multipoles}

We show in Fig. C. 2 the cross-correlation matrices of the " $2 \ell_{i}=$ 0 " and " $2 \ell_{j}=2$ " redshift-space correlations. Thus, in the plane $\left\{R_{i}, R_{j}\right\}$ we plot the contour plots of the matrix

$\mathcal{R}_{R_{i}, R_{j}}^{(s)}=\frac{C_{R_{i}, 2 \ell_{i}=0 ; R_{j}, 2 \ell_{j}=2}^{(s)}}{\sqrt{C_{R_{i}, 0 ; R_{i}, 0}^{(s)} C_{R_{j}, 2 ; R_{j}, 2}^{(s)}}}$.

Because $\ell_{i} \neq \ell_{j}$ this matrix is no longer symmetric in the plane $\left\{R_{i}, R_{j}\right\}$ and it can be negative for $R_{i}=R_{j}$. We can see that its amplitude is rather small, typically on the order of 0.05 , even along the diagonal. Indeed, because $\ell_{i} \neq \ell_{j}$ the pure shot-noise contribution to Eq. (34) vanishes (the factor $\delta_{\ell_{i}, \ell_{j}}\left(4 \ell_{i}+1\right)$ within the brackets). This is due to the orthogonality of the Legendre polynomials, which are the basis of the multipole decomposition of the redshift-space correlation. The small amplitude of $\left\{R_{i}, R_{j}\right\}$ found in Fig. C.2 means that the full covariance matrix $C_{i, j}^{(s)}$ is approximately block-diagonal in the space $\left\{R_{i}, 2 \ell_{i}\right\}$. Therefore, we may neglect $C_{i, j}^{(s)}$ for $\ell_{i} \neq \ell_{j}$ and decouple the analysis of $\xi_{R_{i}, 0}^{(s)}$ and $\xi_{R_{i}, 2}^{(s)}$.

\section{References}

\section{Abell, G. O. 1958, ApJS, 3, 211}

Adami, C., \& Ulmer, M. P. 2000, A\&A, 361, 13

Benjamin, J., Heymans, C., Semboloni, E., et al. 2007, MNRAS, 381, 702

Bernardeau, F., Colombi, S., Gaztañaga, E., \& Scoccimarro, R. 2002a, Phys. Rep., 367, 1

Bernardeau, F., Mellier, Y., \& van Waerbeke, L. 2002b, A\&A, 389, L28 Bernstein, G. M. 1994, ApJ, 424, 569

Blake, C., Davis, T., Poole, G. B., et al. 2011a, MNRAS, 415, 2892

Blake, C., Glazebrook, K., Davis, T. M., et al. 2011b, MNRAS, 418, 1725

Böhringer, H., Schuecker, P., Guzzo, L., et al. 2001, A\&A, 369, 826

Borgani, S., Rosati, P., Tozzi, P., et al. 2001, ApJ, 561, 13

Burenin, R. A., Vikhlinin, A., Hornstrup, A., et al. 2007, ApJS, 172, 561

Cabré, A., \& Gaztañaga, E. 2009, MNRAS, 396, 1119

Clerc, N., Sadibekova, T., Pierre, M., et al. 2012, MNRAS, in press

Cole, S., Percival, W. J., Peacock, J. A., et al. 2005, MNRAS, 362, 505

Colombi, S., Dodelson, S., \& Widrow, L. M. 1996, ApJ, 458, 1

Coziol, R., Andernach, H., Caretta, C. A., Alamo-Martínez, K. A., \& Tago, E. 2009, AJ, 137, 4795

Desjacques, V., Crocce, M., Scoccimarro, R., \& Sheth, R. K. 2010, Phys. Rev. D, 82,103529

Eisenstein, D. J., \& Hut, P. 1998, ApJ, 498, 137

Eisenstein, D. J., Weinberg, D. H., Agol, E., et al. 2011, AJ, 142, 72

Eisenstein, D. J., Zehavi, I., Hogg, D. W., et al. 2005, ApJ, 633, 560

Fry, J. N. 1984, ApJ, 277, L5

Gradshteyn, I. S., \& Ryzhik, I. M. 1994, Table of integrals, series and products,

eds. I. S. Gradshteyn, \&amp; I. M. Ryzhik (New York: Academic Press)

Groth, E. J., \& Peebles, P. J. E. 1977, AJ, 217, 385

Guzzo, L., Pierleoni, M., Meneux, B., et al. 2008, Nature, 451, 541

Hamilton, A. J. S. 1992, ApJ, 385, L5

Jackson, J. C. 1972, MNRAS, 156, 1P
Kaiser, N. 1987, MNRAS, 227, 1

Kaiser, N. 1992, ApJ, 388, 272

Katgert, P., Mazure, A., Perea, J., et al. 1996, A\&A, 310, 8

Kilbinger, M., Benabed, K., Guy, J., et al. 2009, A\&A, 497, 677

Koester, B. P., McKay, T. A., Annis, J., et al. 2007a, ApJ, 660, 239

Koester, B. P., McKay, T. A., Annis, J., et al. 2007b, ApJ, 660, 221

Komatsu, E., Smith, K. M., Dunkley, J., et al. 2011, ApJS, 192, 18

Landy, S. D., \& Szalay, A. S. 1993, ApJ, 412, 64

Limber, D. N. 1953, ApJ, 117, 134

Manera, M., \& Gaztanaga, E. 2011, MNRAS, 415, 383

Mantz, A., Allen, S. W., Rapetti, D., \& Ebeling, H. 2010, MNRAS, 406, 1759

Massey, R., Rhodes, J., Leauthaud, A., et al. 2007, ApJS, 172, 239

Mehrtens, N., Romer, A. K., Lloyd-Davies, E. J., et al. 2012, MNRAS, 423, 1024

Meiksin, A., \& White, M. 1999, MNRAS, 308, 1179

Melin, J.-B., Bartlett, J. G., \& Delabrouille, J. 2006, A\&A, 459, 341

Moles, M., Benítez, N., Aguerri, J. A. L., et al. 2008, AJ, 136, 1325

Munshi, D., Valageas, P., van Waerbeke, L., \& Heavens, A. 2008, Phys. Rep., 462, 67

Nishimichi, T., \& Taruya, A. 2011, Phys. Rev. D, 84, 043526

Oegerle, W. R., \& Hill, J. M. 2001, AJ, 122, 2858

Pacaud, F., Pierre, M., Adami, C., et al. 2007, MNRAS, 382, 1289

Pacaud, F., Pierre, M., Refregier, A., et al. 2006, MNRAS, 372, 578

Peacock, J. A., Cole, S., Norberg, P., et al. 2001, Nature, 410, 169

Peebles, P. J. E. 1980, The large-scale structure of the universe, ed. P. J. E. Peebles, (Princeton, N.J., USA:Princeton University Press)

Peebles, P. J. E., \& Hauser, M. G. 1974, ApJS, 28, 19

Percival, W. J., \& White, M. 2009, MNRAS, 393, 297

Pierre, M., Pacaud, F., Juin, J. B., et al. 2011, MNRAS, 414, 1732

Pillepich, A., Porciani, C., \& Reiprich, T. H. 2012, MNRAS, 422, 44

Planck Collaboration 2011, A\&A, 536, A8

Predehl, P., Boehringer, H., \& et al., H. B. 2009, Proc. Conf. X-ray Astronomy 2009, Bologna, September 2009

Refregier, A., Amara, A., Kitching, T. D., \& Rassat, A. 2011, A\&A, 528, A33

Reichardt, C. L., Stalder, B., Bleem, L. E., et al. 2012, ApJ, submitted [arXiv: 1203.5775]

Reid, B. A., \& White, M. 2011, MNRAS, 417, 1913

Reid, B. A., Samushia, L., White, M., et al. 2012, MNRAS, submitted [arXiv: 1203.6641]

Ross, N. P., da Ângela, J., Shanks, T., et al. 2007, MNRAS, 381, 573

Ross, A. J., Percival, W. J., Crocce, M., Cabré, A., \& Gaztañaga, E. 2011, MNRAS, 415, 2193

Rozo, E., Wechsler, R. H., Rykoff, E. S., et al. 2010, ApJ, 708, 645

Sarazin, C. L. 1988, X-ray emission from clusters of galaxies

Schrabback, T., Hartlap, J., Joachimi, B., et al. 2010, A\&A, 516, A63

Sehgal, N., Trac, H., Acquaviva, V., et al. 2011, ApJ, 732, 44

Slosar, A., Font-Ribera, A., Pieri, M. M., et al. 2011, J. Cosmology Astropart. Phys., 9, 1

Smith, R. E., Peacock, J. A., Jenkins, A., et al. 2003, MNRAS, 341, 1311

Spergel, D. N., Bean, R., Doré, O., et al. 2007, ApJS, 170, 377

Sunyaev, R. A., \& Zeldovich, Y. B. 1972, Comments on Astrophysics and Space Physics, 4, 173

Szapudi, I. 2001, in Annals of the New York Academy of Sciences, The Onset of Nonlinearity in Cosmology, ed. J. N. Fry, J. R. Buchler, \& H. Kandrup, 927, 94

Szapudi, I., \& Colombi, S. 1996, ApJ, 470, 131

Taruya, A., Nishimichi, T., \& Saito, S. 2010, Phys. Rev. D, 82, 063522

Teyssier, R. 2002, A\&A, 385, 337

Teyssier, R., Pires, S., Prunet, S., et al. 2009, A\&A, 497, 335

Tinker, J., Kravtsov, A. V., Klypin, A., et al. 2008, ApJ, 688, 709

Tinker, J. L., Robertson, B. E., Kravtsov, A. V., et al. 2010, ApJ, 724, 878

Valageas, P., Clerc, N., Pacaud, F., \& Pierre, M. 2011, A\&A, 536, A95

Vanderlinde, K., Crawford, T. M., de Haan, T., et al. 2010, ApJ, 722, 1180 